\title{
Auf dem Weg zur Beteiligungsgesellschaft ${ }^{1}$
}

GREGOR HUSI

\section{EINLEITUNG}

\author{
Dene wos guet geit \\ Giengs besser \\ Giengs dene besser \\ Wos weniger guet geit \\ Was aber nid geit \\ Ohni dass's dene \\ Weniger guet geit \\ Wos guet geit \\ Drum geit weni \\ Für dass es dene \\ Besser geit \\ Wos weniger guet geit \\ Und drum geits o \\ Dene nid besser \\ Wos guet geit \\ MANI MATTER
}

»Freiheit, Gleichheit, Brüderlichkeit« - keine politische Formel hat je wieder dieselbe Bekanntheit erlangt wie jene Wertedreiheit aus der Zeit der Französischen Revolution. Freilich, wandelt man auf den Spuren politischer Werte im 18. und 19. Jahrhundert (Husi/Meier Kressig 1998: 148-158), stößt man auf viele andere Werte-Sammlungen, darunter oft Dreiheiten. Eine wichtige, historisch belegbare These lautet, dass Sicherheit - und nicht primär die »Fraternität« - gleichgewichtig neben Freiheit und Gleichheit die politische Moderne grundiert hat (Husi/Meier Kressig 1998: 158ff.). »Die Überzeugung, Sicherheit gehöre zusammen mit Freiheit und Gleichheit zur normativen Grundlage politischer Ordnung, entspricht dem Selbstverständ-

1 Für kritische Kommentare zu diesem Text danke ich ganz besonders Bruno Keller, Marcel Meier Kressig, Peter A. Schmid und Beatrice Windlin. 
nis der demokratischen Moderne «, hält Marti (2006: 105) fest. In dieser Moderne entwickelt sich das Bedeutungsfeld von Sicherheit und Unsicherheit, Risiko und Gefahr, Gewissheit und Schutz zunächst im politischen Diskurs und daraufhin auch in anderen Bereichen. Diese Entwicklung und der entsprechende Bedeutungszuwachs haben sogar dazu geführt, dass Menschen heutiger westlicher Demokratien in einer "Sicherheitsgesellschaft» leben, »die sich dadurch auszeichnet, dass Regieren zunehmend über Verunsicherung erfolgt und das Streben nach umfassender Sicherheit anderen Zielvorgaben vollständig übergeordnet und zum Wert an sich wird « (Singelnstein/Stolle 2006: 13). Begriffe wie »Gleichheitsgesellschaft« oder »Freiheitsgesellschaft« haben sich demgegenüber noch nicht gebildet. Werte - so auch Sicherheit - entfalten ihre Geltung nur im Kontext von und zuweilen in Konkurrenz mit anderen Werten. Grund genug, über Grundwerte von Demokratien nachzudenken und deren Platz in einer gesellschaftstheoretischen Konzeption zu bestimmen.

Jener Denker, der das Fundament der - zumindest soziologischen - Reflexion von Werten legte, ist zweifellos Max Weber. Er verfolgte genau, wie der Geist des Kapitalismus menschliches Zusammenleben revolutionierte und schuf in seiner Handlungstheorie mit »wertrationalem Handeln« einen besonderen, in Werten gründenden Handlungstypus. Weber schenkte, verglichen mit dem Kapitalismus, dem »Demokratismus« weniger Aufmerksamkeit, wie man, in positiver Bedeutung, spätestens seit Friedrich Schlegels Versuch über den Begriff des Republikanismus von 1796 oder auch mit Max Scheler sagen kann, der den Demokratismus zu den »Weltanschauungsformen« zählte (Truhlar 2006: 166, 168; vgl. auch Husi/Meier Kressig 1998: 146). Webers geringeres Interesse erstaunt, denn Modernisierungsprozesse im Geist des Demokratismus haben die Moderne nicht minder geprägt und lassen sich mit seiner Konzeption der Verwirklichung von Werten einsichtsreich analysieren: In Webers Theorie (Husi/Meier Kressig 1998: 60-144) eignet Werten eine eigene Wirklichkeit, die Wertewirklichkeit (demokratische Grundwerte), d.h. Menschen verinnerlichen Werte in ihren Lebenszielen und wünschen sich demnach Zustände und Ereignisse in der subjektiven, sozialen und objektiven Welt, die den bevorzugten Werten entsprechen. Dabei entwickeln sie in Bezug auf dieselben Werte zuweilen sehr unterschiedliche Wertinterpretationen. Indem sich Menschen in ihrem Handeln an interpretierten Werten orientieren und ihre Lebensweise an ihnen ausrichten, tragen sie, mehr oder weniger erfolgreich, zum Prozess der Werteverwirklichung (Demokratisierung) bei, die stets vorläufige Ergebnisse, die Werteverwirklichtheit (Demokratiediagnose), zeitigt.

Der nachfolgende Beitrag versucht, diese Zusammenhänge zu erschließen, und zwar auf der Grundlage einer handlungstheoretisch verankerten Gesellschaftstheorie. Zunächst wird aufgezeigt, welche Grundwerte den Geist des Demokratismus prägen. Daraufhin werden Demokratiediagnosen gesichtet, um die >Verwirklichtheit demokratischer Werte einzuschätzen. Diese Einschätzungen reichern zugleich die Auffassung von Demokratie 
und Demokratisierung an. Danach wird ein differenziertes Gesellschaftsbild in der Nachfolge von Giddens und Bourdieu, die Modale Strukturierungstheorie, skizziert. Es erlaubt, die gesellschaftlichen Orte zu bestimmen, wo die Grundwerte ihre Verwirklichung finden. Indem Prozesse zugleich radikaler und pluraler Demokratisierung in diesem theoretischen Licht betrachtet werden, zeichnen sich zum Schluss die Umrisse einer neuen Leitvorstellung ab; es handelt sich um die Beteiligungsgesellschaft.

\section{Der Geist des Demokratismus: Grundwerte DER MODERNE}

»Wie Sterne am Himmel gibt es aber unzählige Werte, weshalb man Grundwerte braucht, um Emphase auszudrücken. Hier werden dann Traditionsbegriffe wie Freiheit, Gleichheit, Gerechtigkeit, Frieden, Sicherheit, Würde, Wohlfahrt, Solidarität benutzt, um Sonderrang zu markieren. Damit überhöht sich die Ordnung der Wertreferenzen selbst noch einmal.« Luhmann (1993: 19) zählt hier einige Werte auf, die er näher als Grundwerte charakterisiert, ohne sich allerdings auf einen allseits akzeptierten Kanon von Grundwerten beziehen zu können. Welche Werte empfehlen sich denn in systematischer Betrachtung als sogenannte Grundwerte? Im September 2000 trafen sich am Sitz der Vereinten Nationen in New York rund 150 Staats- und Regierungschefs. Sie erörterten die gemeinsamen Herausforderungen der kommenden Jahrzehnte. Es war das größte Treffen von Staatsund Regierungsoberhäuptern in der Geschichte überhaupt. Aus diesem Treffen resultierte die Millenniums-Erklärung der Vereinten Nationen (Generalversammlungsresolution 55/2). Hierin bekräftigen die Teilnehmenden ihre »Verpflichtung auf die Grundsätze der Charta der Vereinten Nationen, die sich als zeitlos und universal bewiesen haben« (Vereinte Nationen 2000: 5). Die Globalisierung solle eine "positive Kraft für alle Menschen der Welt« werden. Die internationalen Beziehungen sollen im 21. Jahrhundert ausdrücklich von folgenden »Grundwerten« geprägt sein:

- Freiheit (Freiheit von: Hunger, Furcht vor Gewalt, Unterdrückung, Ungerechtigkeit)

- Gleichheit (Gleichberechtigung, Chancengleichheit, insbesondere von Männern und Frauen)

- Solidarität (Hilfe der größten Nutznießer an Leidende und Benachteiligte)

- Toleranz (wechselseitige Achtung hinsichtlich Glauben, Kultur und Sprache; Dialog)

- Achtung vor der Natur (nachhaltige Entwicklung bei der Bewirtschaftung lebender Arten und natürlicher Ressourcen im Interesse der Nachfahren) 
- Gemeinsam getragene Verantwortung (Verantwortung für die Gestaltung der weltweiten Entwicklung und die Bewältigung von Bedrohungen des Weltfriedens und der internationalen Sicherheit).

Die ersten vier aufgelisteten Grundwerte beziehen sich allesamt auf menschliches Zusammenleben, während der fünfte Wert der Nachhaltigkeit auf das menschliche Naturverhältnis abzielt. Der sechste und letzte Punkt bricht mit der Logik der vorangehenden, indem er zunächst nicht das letztlich zu Verwirklichende, sondern die Verwirklichenden thematisiert und sie zur Verantwortung zieht. Freilich werden darin mit Frieden und Sicherheit gleich zwei weitere wichtige, das Zusammenleben betreffende Grundwerte angesprochen, die im Text unmittelbar danach unter dem Titel »Frieden, Sicherheit und Abrüstung" separat abgehandelt werden. Der Millenniumserklärung der Vereinten Nationen lassen sich also sechs Grundwerte entnehmen, die sich auf menschliches Zusammenleben, auf Menschen in Gesellschaft beziehen: Freiheit, Gleichheit, Solidarität, Toleranz, Frieden, Sicherheit.

Wie gesagt betrachten die Verfasser der Erklärung selber diese Grundwerte offenbar als zeitlos und universal gültig. Es gibt keinen anderen aktuellen und einflussreicheren Text, der einen Kanon der Grundwerte enthält. Im Folgenden sei darum von den genannten sechs Grundwerten ausgegangen und versucht, sie zu systematisieren. Ganz ähnlich werden die Grundwerte Europas in einer neuen Buchreihe bestimmt (vgl. Sedmak 2010). Hier werden sieben Werte genannt, fünf Werte sind identisch, es fehlt aber Sicherheit, dafür werden Menschenwürde und Gerechtigkeit aufgeführt. Da sich Gerechtigkeit auf Freiheit, Gleichheit und Sicherheit beziehen lässt, besteht die wesentliche Differenz also bei Sicherheit und Menschenwürde.

Um eine erste Ordnung in diese Grundwerte zu bringen, ist eine Unterscheidung von Milton Rokeach (1973: 7ff.) hilfreich. Er unterscheidet terminale und instrumentale Werte. Bei ersteren handelt es sich um Letztwerte, die auf Endzustände menschlicher Existenz abzielen, letztere stehen in deren Dienst und beziehen sich auf nützliche Mittel und Handlungen. Rokeach nennt sie daher auch Mittel-Werte (means-values) und End-Werte (endsvalues). In seinen empirisch gefundenen Werteaufzählungen finden sich Freiheit, Gleichheit, Sicherheit und Frieden explizit bei den terminalen Werten, Toleranz und Solidarität (»hilfreich«) bei den instrumentalen (ebd.: 28). An dieser Sichtweise ist allerdings eine kleine Korrektur anzubringen, denn tatsächlich scheint die Verwirklichung dreier Werte vor allem der Verwirklichung dreier anderer Werte zuzuarbeiten. Es bestehen nämlich folgende hauptsächliche Relationen:

- Förderung von Gleichheit durch Solidarität: Solidarisierungen gleichen an

- Förderung von Freiheit durch Toleranz: Tolerierungen befreien

- Förderung von Sicherheit durch Frieden: Befriedungen sichern. 
Anders gesagt: Solidarisches Handeln macht gleich, tolerantes Handeln macht frei, friedliches Handeln macht sicher - je toleranter, desto freier; je solidarischer, desto >gleicher<; je friedlicher, desto sicherer. Daraus ist zu folgern: Freiheit, Gleichheit und Sicherheit können als primäre und Toleranz, Solidarität und Frieden als sekundäre Grundwerte gelten. Tolerantes, solidarisches, friedliches Handeln lässt Freiheit, Gleichheit und Sicherheit unter Menschen wirklich werden. Bestätigt wird diese Auffassung durch Offe, der einen »Kern von weitgehend unkontroversen Qualitätskriterien« ausmacht, mit denen politische Strukturen und Prozesse auf ihren demokratischen Anspruch hin beurteilt werden:

»Dieser [...] weithin unstrittige Kern setzt sich zusammen aus den Grundsätzen der bürgerlichen Freiheit, der politischen Gleichheit und der zugleich effektiven und verantwortlichen Regierung, welche die zwar nicht spezifisch demokratischen, aber in Demokratien besonders wirksam einklagbaren Staatsfunktionen, nämlich die Erfüllung der Ansprüche der Bürger auf Daseinsvorsorge, (Rechts-)Schutz und (militärische wie soziale, technische wie zivile) Sicherheit, zu erfüllen beauftragt ist. Diese Grundsätze der Freiheit (als Schutz vor der Staatsgewalt, bewirkt durch Grundrechte, Rechtsstaatlichkeit und Gewaltenteilung), der politischen Gleichheit (als faire und egalitäre Mitwirkung an der Ausübung der Staatsgewalt) und der verantwortlichen und effektiven Gewährleistung von Sicherheit (als universalistische Teilhabe an den Leistungen der Staatsgewalt) sind dieselben, die sich als das kumulative Ergebnis der politischen Modernisierungsprozesse im Westen im 18. bis 20. Jahrhundert herausgebildet haben (vgl. T.H. Marshall) und die sich in der Verfassungsformel vom freiheitlichen und demokratischen Sozialstaat wiederfinden.«(Offe 2003: 12f.)

Offe thematisiert hier also dieselbe Wertetriade als primäres demokratisches Richtmaß: Freiheit, Gleichheit, Sicherheit, während Marshall (1992) analysiert, wie bürgerliche, politische und soziale Rechte historisch aufeinander folgten:

»Das bürgerliche Element besteht aus jenen Rechten, die notwendig sind, die individuelle Freiheit zu sichern [...] Mit dem politischen Element bezeichne ich das Recht auf die Teilnahme am Gebrauch politischer Macht [...] Mit dem sozialen Element bezeichne ich eine ganze Reihe von Rechten, vom Recht auf ein Mindestmass an wirtschaftlicher Wohlfahrt und Sicherheit, über das Recht an einem vollen Anteil am gesellschaftlichen Erbe, bis zum Recht auf ein Leben als zivilisiertes Wesen.« (Marshall 1992: 40)

Habermas seinerseits zeichnet diese historische Verrechtlichung in vier Schritten nach (1981: 524-547) und formuliert im Anschluss daran die kritische »These der inneren Kolonialisierung« (ebd.: 539), wonach die kapitalistische Wachstumsdynamik Wirtschaft und Staat immer mehr in die Lebenswelt der Menschen eindringen lässt. Als Gegenmittel sieht Habermas vor: 
»Es geht darum, Lebensbereiche, die funktional notwendig auf eine soziale Integration über Werte, Normen und Verständigungsprozesse angewiesen sind, davor zu bewahren, den Systemimperativen der eigendynamisch wachsenden Subsysteme Wirtschaft und Verwaltung zu verfallen und über das Steuerungsmedium Recht auf ein Prinzip der Vergesellschaftung umgestellt zu werden, das für sie dysfunktional ist.« (ebd.: 547)

Habermas setzt auf »diskursive Willensbildungsprozesse und konsensorientierte Verhandlungs- und Entscheidungsverfahren« (ebd.: 544); sie entsprechen verständigungsorientiertem Handeln. Ambivalenz kennzeichnet denn auch die Sozialpolitik, welche die Umsetzung der von Marshall thematisierten sozialen Rechte gestaltet. Ambivalent erscheint dabei die Verwirklichung der drei genannten primären Grundwerte: Die Sozialpolitik »eröffnet Freiheiten - und schränkt Optionen ein; sie schafft mehr Gleichheit - und neue Ungleichheiten; sie produziert mehr Sicherheit und - eben dadurch immer neue Unsicherheiten. Sie verwandelt unüberschaubare Gefährdungen in kalkulierbare Risiken - und diese im Zweifel wieder zurück in Gefahren.« (Lessenich 2006: 557)

An Marshalls Analyse knüpft auch Talcott Parsons (1970: 105) an, der entschiedener als viele andere - zunächst - die industrielle Revolution und die demokratische Revolution gleichgewichtig in seine theoretischen Überlegungen zur Entwicklung des »Systems moderner Gesellschaften« einbezieht (ebd.: 96-109).

»Die demokratische Revolution umfasste in erster Linie den integrativen Aspekt der Gesellschaften; sie konzentrierte sich auf die politische Bedeutung der Mitgliedschaft in der gesellschaftlichen Gemeinschaft und damit auf die Rechtfertigung von Ungleichheiten des Wohlstands sowie, was wichtiger ist, der politischen Autorität und sozialer Privilegien.« (ebd.: 97)

Hinzu kommt später die »Bildungsrevolution«, welche »die Themen der industriellen und der demokratischen Revolution, Chancengleichheit und Gleichheit als Bürger, miteinander verbindet« (ebd.: 123) und Parsons »genauso wichtig« (ebd.: 120) wie die beiden vorangegangenen Revolutionen dünkt.

Sämtliche sechs erwähnten Grundwerte haben sich mit der demokratischen Revolution allgemein durchzusetzen begonnen, mit der politischen Moderne, die die swestlichen Nationen hunderts erfasste. Seither entwickeln sich zunächst nur wenige betroffene und im Laufe der Zeit immer mehr Länder im Geiste des Demokratismus. Von Samuel P. Huntington stammt der Ausdruck der Demokratisierungswelle. Laut Huntington (1991: 13-26) setzte die erste dieser Wellen im Amerika des frühen 19. Jahrhunderts ein, dauerte bis zum Ende des Ersten Weltkriegs und führte zu rund 30 entwickelten demokratischen Staaten. Die zweite initiierten die Alliierten nach dem Ende des Zweiten Weltkriegs, sie 
endete mit der Entkolonialisierung in den 1950er und 1960er Jahren. Die dritte begann in den 1970er Jahren vor allem in Südosteuropa und Lateinamerika. Diese Periodisierung Huntingtons wird durch Klaus von Beyme (1994: 11f.) ergänzt, der eine vierte Welle in den späten 1980er und frühen 1990er Jahren Ost- und Mitteleuropa erfassen sieht (und laut dem, von Huntingtons Periodisierung abweichend, die erste Welle nach dem Ersten Weltkrieg einsetzte). Eine fünfte Welle kündigte sich im Jahre 2011 in der arabischen Welt an (vgl. Perthes 2011). »Die Demokratie ist wahrscheinlich die mächtigste und zündendste Idee des zwanzigsten Jahrhunderts«, vermutet denn Giddens (2001: 88). Damit stellt sich die Frage, welche Diagnose der Verwirklichung der Demokratie heute gestellt werden kann.

\section{DEMOKRATIEDIAGNOSEN}

Allerdings kann nicht wirklich von der Verwirklichung der Demokratie in der Einzahl gesprochen werden:

»Demokratien treten in einer Vielzahl von Strukturvarianten auf. Sie können präsidentiell oder parlamentarisch verfasst sein, sich auf eine unitarische oder föderale Staatsform beziehen, mit dem Mehrheits- oder dem Verhältniswahlrecht operieren, ein Zwei- oder ein Mehrparteiensystem aufweisen, nur repräsentative oder zusätzlich auch direktdemokratische (`plebiszitäre ) Beteiligungsformen zur Verfügung stellen, zivilgesellschaftlichen und intermediären Assoziationen mehr oder weniger Raum geben, ein höheres oder ein geringeres Maß der Gewaltenteilung zwischen Parlament und Regierung aufweisen und Ein- oder Mehrkammersysteme sein. Keine Demokratie ist ihrer institutionellen Struktur nach eine Kopie irgendeiner anderen.« (Offe 2003: 9)

Offe stellt, noch ganz allgemein, die Diagnose einer »eher abnehmenden demokratischen Inklusion« (ebd.: 14), um sie sodann zu differenzieren: »Nicht nur sind die neuen Demokratien >noch nicht vermeintlich konsolidierten alten; auch diese sind ınicht mehr das, was sie ihrem Anspruch und Selbstverständnis nach einmal waren oder zu sein wähnten.« (ebd.: 10)

Im Diskurs über die heutige Verwirklichung der Demokratie finden sich einige prägnante Positionen: (a) Jean-Marie Guéhenno (1994) formuliert die These, wir seien am »Ende der Demokratie« angelangt. (b) Colin Crouch (2008) sieht Demokratien auf eine »Postdemokratie« zusteuern. Diesen beiden, in griffige Formeln gebrachten Diagnosen stehen jedoch andere Beobachtungen gegenüber. (c) Claus Offe (2003) nimmt mit anderen eine »Demokratisierung der Demokratie« wahr, (d) Oskar Negt erkennt die »Demokratie als Lebensform«, und ähnlich weisen (e) Ernesto Laclau und Chantal Mouffe (1991) auf eine »Radikale Demokratie« hin. Alle drei letzt- 
genannten Einschätzungen enthalten keineswegs bloß normative Postulate. Was besagen die fünf Diagnosen genau?

(a) Guéhenno umreißt das Ende der Demokratie mit Verweis auf ein folgenreiches Jahr: »Das Jahr 1989 bezeichnet nicht das Ende einer Epoche, die 1945 oder 1917 begonnen hätte, sondern das Ende dessen, was durch die Revolution von 1789 institutionalisiert wurde. Mit diesem Jahr endet das Zeitalter der Nationalstaaten.« (1994: 10) Guéhenno kontrastiert ein vergangenes »institutionelles Zeitalter«, das auf den Ideen der Aufklärungsphilosophen beruht, und ein künftiges »imperiales Zeitalter«. »Heute muss man sich fragen, ob es eine Demokratie ohne Nation geben kann.« (ebd.: 12) Guéhenno malt folgendes Zukunftsbild:

»Die menschliche Gemeinschaft ist zu groß geworden, um noch ein politisches Gemeinwesen zu bilden. Die Bürger stellen immer weniger eine Gesamtheit dar, in der kollektive Souveränität zum Ausdruck kommen könnte; sie sind lediglich juristische Personen mit Rechten und Pflichten, sie befinden sich in einem abstrakten Raum mit zunehmend ungewissen territorialen Grenzen.« (ebd.: 13)

Der imperiale Charakter zeige sich überdies daran, dass das europäische Politikverständnis einem asiatischen weiche. Dieses Imperium sei »eine Welt, die gleichzeitig geeint und ohne Zentrum ist« (ebd.). Im kommenden »Zeitalter der Komplexität« könne kein Zentrum mehr auf eine »pyramidale Machtstruktur« bauen. Für Guéhenno war denn die »nationalstaatliche Epoche« nur eine »Episode der Menschheitsgeschichte«. Allerdings leben wir in einer Zeit des Übergangs:

»Wir befinden uns in der vorgeschichtlichen Phase dieses neuen Zeitalters, und die Logik der Nationalstaaten wird noch lange neben der Logik einer imperialen Welt gelten, von der man nicht weiß, ob sie, wie die Imperien, die ihr vorausgingen, ihre Macht auszudehnen beabsichtigt, um die aus dem Umfeld drohenden Gefahren zu mindern, oder ob sie durch ihre eigene Logik gelähmt sein wird. [...] Was wird geschehen, wenn die Diffusion der Macht in dieser Welt zu vollkommen geworden ist, als dass sich ein politischer Wille bilden könnte? (ebd.: 172f.)

(b) Die These der Postdemokratie wird insbesondere von Colin Crouch vertreten. Dieser macht einen Widerspruch aus: Formal betrachtet scheinen heutige Demokratien durchaus intakt. Der Unterschied zu prädemokratischen politischen Verhältnissen zeigt sich nämlich an Parteienkonkurrenz, Wahlkämpfen, Wahlen und allfälligen Abstimmungen sowie Gewaltenteilung. Aber das Volk partizipiert kaum mehr wie vorgesehen am politischen Geschehen, und das mindert dessen Legitimation. Wahlkämpfe gehen als medial inszenierte, manipulative Spektakel über die Bühne, in denen Experten diskutierte Themen bestimmen, während der politische Prozess durch einflussreiche Lobbyarbeit hinter geschlossenen Türen korrumpiert wird. »Die Mehrheit der Bürger spielt dabei eine passive, schweigende, ja sogar 
apathische Rolle.« (Crouch 2008: 10) Dies bezeichnet Crouch mit dem Begriff der Postdemokratie, der dafür steht, dass »wir gleichsam am anderen Ende der Parabel der Demokratie angekommen sind « (ebd.: 30). Crouch gibt sich »davon überzeugt, dass wir uns dem postdemokratischen Pol immer mehr annähern« (ebd.: 11).

»Der Begriff Postdemokratie kann uns dabei helfen, Situationen zu beschreiben, in denen sich nach einem Augenblick der Demokratie Langeweile, Frustration und Desillusionierung breitgemacht haben; in denen die Repräsentanten mächtiger Interessengruppen, die nur für eine kleine Minderheit sprechen, weit aktiver sind als die Mehrheit der Bürger, wenn es darum geht, das politische System für die eigenen Ziele einzuspannen; in denen politische Eliten gelernt haben, die Forderungen der Menschen zu lenken und zu manipulieren; in denen man die Bürger durch Werbekampagnen >von oben` dazu überreden muss, überhaupt zur Wahl zu gehen.« (ebd.: 30)

Womit wäre dem zu begegnen? »Erstens mit Maßnahmen, die darauf zielen, die wachsende Dominanz der ökonomischen Eliten zu begrenzen; zweitens mit Reformen der politischen Praxis als solcher; und drittens gibt es Handlungsmöglichkeiten, die den Bürgern selbst offenstehen.« (Crouch 2008: 133) Jörke (2005) folgt Crouchs These, setzt die Akzente indes etwas anders. Er bezweifelt die Möglichkeiten moderner Demokratien, besonders zwei Versprechen einzulösen: das Versprechen der politischen Gleichheit, wonach allen Bürgerinnen und Bürgern gleichermaßen Wahlbeteiligung, politische Karriere und Interessenverfolgung möglich sein sollten - vor allem für untere Schichten trifft dies nicht $\mathrm{zu}$; und das Versprechen des lebensweltlichen Einflusses, wonach Betroffene den Lauf der Dinge in der eigenen Lebenswelt nach eigenem Gutdünken mitgestalten können sollten die dafür nötigen politischen Handlungsspielräume werden allzu sehr beschnitten durch Entscheidungen, die an gewählten Parlamenten weitgehend vorbei, aufgrund von Verhandlungen zwischen Exekutiven und potenten gesellschaftlichen Kräften (»Postparlamentarismus«) getroffen werden, durch supranationale Organisationen (z.B. Weltbank, Internationaler Währungsfonds, Welthandelsorganisation) und durch die internationalisierte Ökonomie, die Regierungen zu einem globalen Standortwettbewerb um ein "günstiges Investitionsklima« verleitet.

Angesichts solcher Vermutungen haben die Universitäten Zürich und Bern sowie das Wissenschaftszentrum Berlin gemeinsam ein Demokratiebarometer entwickelt, das Qualitätsunterschiede von Demokratien messen helfen soll. Dem Messinstrument liegen drei »konstituierende Prinzipien« zugrunde: Freiheit, Gleichheit und Kontrolle. Im Aspekt der Kontrolle verbergen sich Aspekte des Werts Sicherheit. Aus diesen drei Prinzipien werden neun "grundlegende Funktionen« abgeleitet, die zu 18 Komponenten, 51 Subkomponenten und schließlich 100 Indikatoren weiter differenziert werden. Zur Konzeption werden drei Demokratieverständnisse unterschieden: Die liberale Demokratie (Locke, Montesquieu, Mill, Tocqueville) 
ist ein Mittel, um die Freiheitsrechte der Bürger vor staatlichen Übergriffen zu bewahren; freie und faire Wahlen lassen politische Eliten bestimmen, denen aufgegeben wird, Interessen wahrzunehmen. Die partizipatorische Demokratie (Rousseau) gewährt Bürgern möglichst viele Gelegenheiten zur Beteiligung und Mitsprache, um individuelle Selbstentfaltung, politisches Interesse und gesellschaftliche Integration $\mathrm{zu}$ fördern und politische Entscheide breit abzustützen. Die soziale Demokratie (Rawls, Sen) bezieht Voraussetzungen ein, die für die Realisierung der beiden obigen Demokratiemodelle, besonders für die Verwirklichung freier und fairer Partizipation, erfüllt sein müssen, gewährt deshalb Sozialrechte und bekämpft soziale Ungleichheiten. Eine neuere »Theorie der Sozialen Demokratie« entwirft z.B. Thomas Meyer, indem er »Formalgeltung« und »Realwirkung« konfrontiert, d.h. nicht nur die Legitimität und Gestalt politischer Institutionen betrachtet, sondern eine »Analyse der empirischen Voraussetzungen für die Entfaltung ihrer realen Wirksamkeit« (2005: 11) vornimmt. Zur »förmlichen Rechtsgeltung« von Grundrechten kommt »die tatsächliche Verfügung einer Person über die Chancen zur Nutzung ihrer Grundrechte« (ebd.: 12) hinzu. Meyer knüpft insbesondere an die Arbeiten von Hermann Heller an. So

»basiert die Soziale Demokratie auf der Vorstellung, dass Demokratie in Verbindung mit den Grundrechten im gesellschaftlichen und wirtschaftlichen Bereich einer ihnen gerecht werdenden Verfassung der geregelten Teilhabe, des Rechtsanspruchs auf soziale Sicherung und der gerechtigkeitsorientierten Distribution sowie einer diesen Werten verpflichteten regulativen und distributiven Politik des demokratischen Staates bedarf.« (ebd.: 13)

Das Demokratiebarometer basiert auf einem »Demokratiekonzept mittlerer Reichweite « und kombiniert Elemente des liberalen und partizipatorischen Modells. Es lässt das dritte Modell explizit außen vor, da es Outputs als genuin politische, durch Demokratie als Mittel erzeugte Entscheidungen betrachtet (und nicht als Bestandteil von Demokratie) und da Politikergebnisse im Sinne von Outcomes nicht nur aus demokratischen Entscheidungen resultieren, sondern auch aus anderen gesellschaftlichen (z.B. wirtschaftlichen) Faktoren hervorgehen. »Ausgangspunkt bildet die Prämisse, dass demokratische Systeme eine Balance zwischen den interdependenten Werten >Freiheit` und >Gleichheit` herzustellen versuchen und sich dazu einer dritten demokratieinhärenten Dimension bedienen: Kontrolle.« (Bühlmann/ Merkel/Müller/Giebler/Weßels 2011: 4) 
Abbildung 1: Qualitätselemente der Demokratie

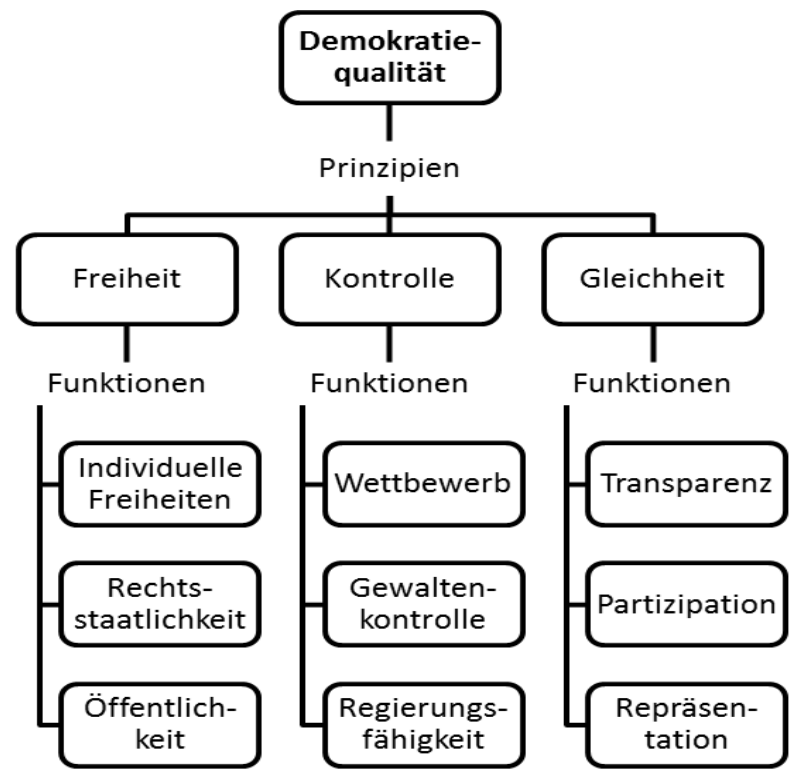

Quelle: Bühlmann/Merkel/Müller/Giebler/Weßels 2011: 9

Gleiches im Sinn hat zuvor bereits das Konzept der »eingebetteten Demokratie« (Merkel 2003), das dem »komplexen Institutionengefüge« moderner Demokratien gerecht werden und deren »Defekte« erkennen will. Es besteht aus fünf Teilregimes. Im Zentrum steht (1) das »Wahlregime«: periodische, freie, allgemeine, gleiche und faire Wahlen. Zusammen mit (2) den »politischen Partizipationsrechten « (Recht auf Meinungs- und Redefreiheit, Assoziations-, Demonstrations-, Petitionsrecht), welche die Arena der Öffentlichkeit konstituieren, bilden sie die »vertikale Demokratiedimension" (»vertikale Verantwortlichkeit«), insofern »die sharte〈 periodische Kontrolle durch Wahlen durch die >weiche`, aber stetige Kontrolle der Öffentlichkeit zwischen den Wahlen ergänzt wird« (ebd.: 50). (3) Die »bürgerlichen Freiheits- und Abwehrrechte« umfassen individuelle Schutzrechte gegenüber dem Staat hinsichtlich Leben, Freiheit, Eigentum sowie den gleichen Rechtszugang und die Gleichbehandlung vor dem Gesetz. (4) Die »horizontale Verantwortlichkeit« wird durch die »Gewaltenteilung« realisiert, die gleichzeitige, wechselseitige Abhängigkeit und Selbständigkeit von Legislative, Exekutive und Judikative. Hinzu kommt schließlich (5) die »effektive Regierungsgewalt«, die tatsächlich von gewählten Repräsentanten (und z.B. nicht vom Militär) ausgeübt werden soll und auch bedeutet, dass es keine »reservierten Politikdomänen« außerhalb ihres Einflusses gibt. 
$»$ Die Dominanz $[\ldots]$ eines der Teilregimes über ein anderes wird erschwert und so die Spannung zwischen Prinzipien der politischen Gleichheit, Freiheit und Kontrolle gemildert. Es ist also gerade die wechselseitige Einbettung der einzelnen Institutionen der Demokratie in ein Gesamtgeflecht institutioneller Teilregimes, die erst die Demokratie funktions- und widerstandsfähig macht.« (ebd.: 56)

Über diese »interne Einbettung« hinaus sieht das Konzept auch eine »externe Einbettung« vor. »Diese Einbettungsringe sind Möglichkeits- oder Unmöglichkeitsbedingungen, sie verbessern oder verschlechtern die Qualität rechtsstaatlicher Demokratie, aber sie sind keine definierenden Bestandteile der Demokratie selbst« (ebd.: 57). Zu ihnen zählen der sozioökonomische Kontext, die Zivilgesellschaft und die internationale Integration: Armut ist zu vermeiden, da sie bei der Wahrnehmung bürgerlicher Schutzrechte und politischer Partizipationsrechte benachteiligt; die Eigenschaften

»der Zivilgesellschaft schützen das Individuum vor staatlicher Willkür (Locke), unterstützen die Herrschaft des Gesetzes und die Balance der Gewalten (Montesquieu), schulen Bürger und rekrutieren politische Eliten (Tocqueville) und institutionalisieren mit dem öffentlichen Raum ein Medium demokratischer Selbstreflexion (Habermas)«(ebd.: 61);

die $»$ Kombination von marktwirtschaftlicher Interessen- und demokratischer Wertegemeinschaft« (z.B. EU), so Merkel, integriert international. Ähnlich gewinnt Vorländer (2003: 94f.) aus der Literatur des abendländischen politischen Denkens sechs Einflüsse, die demokratische Ordnungen stützen helfen, nämlich wechselseitige Bekanntheit durch beschränkte Größe des Gemeinwesens, kulturelle Homogenität, geringe soziale Ungleichheit, die Tugend der Gemeinwohlorientierung, ein geschicktes institutionelles Arrangement sowie eine starke bürgerschaftliche Zivilgesellschaft.

Die Diagnosen des Demokratieendes und der Postdemokratie sowie deren empirische Überprüfung anhand des Demokratiebarometers, wozu die eingebettete Demokratie eine konzeptuelle Grundlage liefert, führen noch nicht sehr weit über den Horizont eines Demokratieverständnisses mit >minimalem Gehalt ' hinaus, den Norberto Bobbio einmal knapp so zusammenfasste: »Garantie der grundlegenden Freiheitsrechte, Existenz mehrerer, miteinander im Wettbewerb stehender Parteien, periodische Wahlen mit allgemeinem Wahlrecht, kollektive Entscheidungen, die entweder [...] im Einvernehmen der Beteiligten oder auf Basis von Mehrheitsentscheidungen getroffen werden.« (1988: 31) Dahinter steht das Interesse an kollektiven Entscheidungen:

$»$ Damit $[\ldots]$ eine von Individuen (einem, wenigen, vielen, allen) gefällte Entscheidung als kollektive Entscheidung akzeptiert werden kann, muss diese Entscheidung auf der Grundlage von Regeln stattfinden (und es spielt keine Rolle, ob es sich dabei um schriftlich fixierte oder um Gewohnheitsregeln handelt), die festlegen, welche 
Individuen dazu berechtigt sind, für alle Gruppenmitglieder verbindlich zu entscheiden, und auf Basis welcher Verfahren dies geschieht.« (ebd.: 8f.).

(c) Für Anthony Giddens (2001: 88) umfasst Demokratie - wie für Bobbio Parteienkonkurrenz, regelmäßige, faire, freie Wahlen sowie Partizipationsund Freiheitsrechte. Er erkennt ein »Paradox der Demokratie«, das darin besteht, »dass sich die Demokratie zwar überall in der Welt ausbreitet, wir jedoch in den alten Demokratien, die der Rest der Welt angeblich nachahmt, eine weitverbreitete Enttäuschung über demokratische Verfahren beobachten« (ebd.: 92). Als Lösung schlägt Giddens vor: »Was wir in den demokratischen Ländern brauchen, ist eine Vertiefung, gleichsam eine Demokratisierung der Demokratie. Außerdem muss die Demokratie die nationalen Grenzen überwinden. Wir brauchen eine Demokratisierung nicht nur innerhalb der Nation, sondern auch über die Ebene der Nation hinaus.« (ebd.: 95) Giddens (ebd.: 96ff.) nennt dazu Stichworte wie Dezentralisierung der Macht, Kampf gegen Korruption, Transparenz, Stärkung der »Zivilkultur« und fordert gar mit einer schönen Wendung den »Aufbau einer Demokratie der Gefühle« (z.B. Toleranz).

Die Beiträge, die Claus Offe (2003) versammelt, lassen sich nun zum Teil gerade als Versuche interpretieren, das - neben dem liberalen und partizipatorischen - erwähnte dritte Modell, die soziale Demokratie, zu erkunden. Sie zehren von den Erfahrungen, welche die politische Moderne mit sich selber gemacht hat, und loten folgerichtig - in begrifflicher Hinsicht zumindest wie Giddens - Möglichkeiten einer Demokratisierung der Demokratie aus. (Auch das Konzept der eingebetteten Demokratie reiht sich hier ein.) »Demokratisierung der Demokratie« ist, so Offe, »im Sinne der Suche nach institutionellen Möglichkeiten zur qualitativen Aufbesserung demokratischer Regierungs- und Verfassungspraxis und ihrer gesellschaftlichen Voraussetzungen« (ebd.: 17) zu verstehen. Dieses Verständnis macht den Blick also auch frei für außerpolitische Bedingungen des Politischen. Das ist ein erster Schritt gleichsam auf die Gesellschaft zu.

(d) Schreitet man in diese Richtung weiter, gelangt man zu einem umfassenderen Begriffsverständnis, nämlich zur Demokratisierung anderer Lebensbereiche als der Politik und ihrer Institutionen. Schmidt (2010: 167) erwähnt denn drei Bedeutungen von »Demokratisierung«: erstens »Bildung einer Demokratie«, zweitens »Erweiterung des demokratischen Prinzips auf zuvor nicht demokratisch organisierte Gesellschaftsbereiche« und drittens »Zunahme des aktiven Anteils der Beherrschten an der Herrschaft«. Nicht nur werden, um zwei zentrale Lebensbereiche, Wirtschaft und Gemeinschaft, als Beispiele zu nehmen, die »Demokratisierung der Marktwirtschaft» (Stein 1995) und eine »Neue Wirtschaftsdemokratie« (Martens 2010) debattiert, sondern ebenso die »Demokratisierung der Geschlechterverhältnisse« (Schäfer 2001) und die »Demokratisierung der Familie« (Beck 1997). 
Der deutsche Bundeskanzler Willy Brandt schlug entschieden diese Richtung ein. »Wir wollen mehr Demokratie wagen« (1979: 252), lautet ein berühmt gewordener Satz aus seiner Regierungserklärung vom 28. Oktober 1969. »Wir stehen nicht am Ende unserer Demokratie, wir fangen erst richtig an« (ebd.: 281), endet die Rede. Brandt suchte nach einer »Alternative zur konservativen und alt-liberalen Auffassung von Demokratie« (1973: 46), einer Alternative, die sich der Rationierung der Demokratie entgegen stellt: »Wir [...] lassen uns auch heute von der Überzeugung leiten, dass die Demokratie nicht auf einen noch so wichtigen Bereich - wie den staatlichen - beschränkt bleiben, dass sie nicht auf Rationen gesetzt werden kann, sondern dass sie das gesamte gesellschaftliche Leben erfassen muss « (ebd.: 46). So prognostiziert Brandt: »Mitbestimmung, Mitverantwortung in den verschiedenen Bereichen unserer Gesellschaft wird eine bewegende Kraft der kommenden Jahre sein« (1979: 252), ohne dass dadurch die »perfekte Demokratie« entstehen könne. Auch für Fritz Vilmar ist denn

$»$ Demokratisierung: Herstellung von Gleichheit und Freiheit in allen Lebensbereichen. Demokratisierung ist also der Inbegriff aller Aktivitäten, deren Ziel es ist, autoritäre Herrschaftsstrukturen zu ersetzen durch Formen der Herrschaftskontrolle von >unten`, der gesellschaftlichen Mitbestimmung, Kooperation und - wo immer möglich - durch freie Selbstbestimmung.« (1973a: 21)

Sie tritt an gegen »ungerechtfertigte Bevormundung und materielle Diskriminierung der Unteren durch die Oberen, Selbstbehauptung der Oligarchien auf Kosten der übrigen Subsystem-Mitglieder und der System-Umwelt« (ebd.: 12). Vilmar (1973b) dokumentiert für seine »Strategie einer gesamtgesellschaftlichen Demokratisierung« Texte zu »gut zwanzig Subsystemen in 6 funktionalen gesellschaftlichen Bereichen« (1973a: 107), und zwar, wie er betont, nicht »zur inhaltlichen, Entscheidungsziele betreffenden Demokratisierung«, sondern bloß zur »Demokratisierung des Entscheidungsprozesses« (ebd.: 11). Subsysteme definiert er als »alle normalerweise unterhalb und innerhalb des staatlichen Gesamtsystems einer Gesellschaft vorfindlichen sozialen Organisationsformen« (ebd.: 428, Anm. 18). Seine »Systematik der gesellschaftlichen Subsysteme« (ebd.: 108f.) gliedert er in folgende Bereiche: primäre Sozialisationssysteme, Bildungssystem, öffentliche Verwaltung, Fürsorgeinstitutionen, Wirtschaft, Verbände und freie Vereinigungen und Volksvertretungen. In einem Schaubild gewichtet Vilmar (ebd.: 111) die 24 unterschiedenen Subsysteme nach Kern-, sekundären und peripheren Bereichen der Demokratisierung. Vilmar knüpft dabei an ein norwegisches Aktionsprogramm an, das unter dem vielsagenden Stichwort Alltagsdemokratie (1973b: 13ff.) Demokratisierungsideen für zahlreiche Lebensbereiche skizziert. In den Blick gelangen auf diese Weise die Lebensbereiche Gemeinschaft, Bildung, Wissenschaft, Medien, Kunst, Verwaltung, Militär, Gesundheit, Soziale Arbeit, Recht, Wirtschaft, Politik und Religion; es fehlen allein Unterhaltung, Sport, Verkehr (Husi 2010: 117f.; vgl. auch 
die Beiträge in Greiffenhagen 1973). Deutlich wird dabei, dass Demokratisierungsprozesse eben auch mikro- und mesogesellschaftlich wirken, d.h. in Gruppen und Organisationen.

Die Auffassung von Demokratie als politischer Institution erweitert sich dadurch, wie Oskar Negt sagt, zur Idee von Demokratie als Lebensform: Nicht nur weitet sich seit einigen Jahrzehnten das Spektrum politisierter Gesellschaftsthemen immer mehr aus; »1968 ist der Aufbruch zu einer Demokratisierung vieler Lebensbereiche« (Negt 2008: 37). Negt hebt somit zwei Aspekte dieser »Basis-Demokratisierung«, der »partizipatorischen Revolution der 1970er Jahre« (Beyme 1994: 9), hervor:

»Zum einen ist es die Politisierung der Interessen und Bedürfnisse der Menschen, sodass sie in einer kritischen Öffentlichkeit in den Prozess politischer Urteilsbildung einbezogen sind. Zum anderen betrifft die Demokratisierung der Gesellschaft, wenn man von Basisdemokratie spricht, die Bereiche konkreten Lebens, welche die alltäglichen Erfahrungen der Menschen bestimmen: in den Betrieben, Büros, Schulen, Universitäten« (Negt 2008: 40)

und - über Wirtschaft, Bildung und Wissenschaft hinaus - noch in anderen Lebensbereichen. Negt erkennt allerdings einen engen Zusammenhang zwischen der Demokratie als politischer Institution und als Lebensform. So stellt er fest, „dass es eine demokratische Gesellschaft ohne Demokraten nicht geben kann. Wenn die Menschen nicht in ihren Alltagsangelegenheiten Mitbestimmungsrechte haben, dann werden die besten demokratischen Institutionen ausgehöhlt« (ebd.); dann werden Menschen »Objekte manipulierender Eliten«. Negt spricht denn folgerichtig von »Demokratie als einer gesellschaftlichen Gesamtverfassung« (ebd.: 41) und lässt kein Missverständnis zu:

»Eine konsequente Demokratisierung aller Lebensbereiche ist die einzige Möglichkeit, ein demokratisches System lebendig zu halten. [...] Eine halbe Demokratie mit freien Wahlen, aber keinerlei Mitbestimmungsrechten in den eigenen, zentralen Lebensbereichen, ist nicht von Dauer. [...] Nur als Lebensform hat Demokratie eine Zukunftschance.« (2010: 514f., Herv. i.O.)

An vorderster Stelle denkt Negt dabei an die »Wirtschaftsdemokratie«. Für Bobbio ist Demokratie ein »Ensemble von Regeln« (1988: 33). Sie ist nämlich »als ein Ensemble von (primären oder Grund-)Regeln zu begreifen, die festlegen, wer zur Teilnahme an den kollektiven Entscheidungen berechtigt ist und mit welchen Verfahren diese Entscheidungen getroffen werden« (ebd.: 8). Das bedeutet: Demokratie findet sich überall da, d.h. in allen Lebensbereichen, in denen sich demokratische Regeln etablieren. Dieses Ensemble von Regeln, dieses Regulativ grundiert, so können wir zusammenfassen, nicht mehr nur die Politik, sondern färbt mehr oder weniger alle Lebensbereiche, die sich in der Moderne ausdifferenziert haben - zumindest 
geraten alle Lebensbereiche unter Demokratisierungsdruck; und die Geltung dieses Ensembles von Regeln hängt von Voraussetzungen ab. Demokratisierung im engeren Sinne ist demnach jener gesellschaftliche Prozess, der die Geltung des demokratischen Ensembles von Regeln in verschiedenen Lebensbereichen institutionalisiert. Und Demokratisierung im weiteren Sinne ist jener gesellschaftliche Prozess, der zugleich unterstützende strukturelle Voraussetzungen dafür schafft. Es geht also um die Institutionalisierung als die Etablierung des demokratischen Ensembles von Regeln wie um die Schaffung gesellschaftlicher Bedingungen, welche die tatsächliche Geltung der Regeln fördern und dabei zweckmäßige Ergebnisse hervorbringen lassen, sodass, wie Schmidt (2010) schreibt, der aktive Anteil der Beherrschten an der Herrschaft zunimmt. Moderne Gesellschaften wandeln sich demnach im engeren und weiteren Sinne im Geiste des Demokratismus, und zwar genau betrachtet auf vier Weisen:

1. Einrichtung der Demokratie als politische Institution

2. Einrichtung förderlicher Voraussetzungen für die politische Mitbestimmung

3. Einrichtung der Demokratie in Form außerpolitischer Institutionen

4. Einrichtung förderlicher Voraussetzungen für die außerpolitische Mitbestimmung.

An dieser Stelle darf Karl Mannheims Begriff der Fundamentaldemokratisierung nicht fehlen. So »aktiviert die industrielle Gesellschaft immer mehr auch diejenigen Schichten und Gruppen, die früher am politischen Leben nur passiv teilnahmen. Ich möchte diese neue weitgehende Aktivierung der Massen die 〉Fundamentaldemokratisierung der Gesellschaft» nennen«, schreibt Mannheim (1958: 51). Fundamental demokratisieren sich moderne Gesellschaften nicht nur in der von Mannheim angesprochenen Sozialdimension, sondern, denkt man über den Rand des Lebensbereichs Politik hinaus sowie an Demokratiebedingungen, auch in der Sachdimension. »Ohne Fundamentaldemokratisierung funktioniert die Demokratie nicht«, bringt Greiffenhagen (1973: 25) den Gedanken auf den Punkt. Zu Beginn des dritten Jahrtausends fragt sich nun, was diese Fundamentaldemokratisierung in der Raumdimension bedeutet. Zur Debatte stehen besonders drei Modelle (Vorländer 2003: 121ff.): Globales Regieren (global governance) verweist auf ein repräsentatives und demokratisches System der Vereinten Nationen, auf eine liberale Demokratie im Weltmaßstab, also auf einen Weltstaat mit einer demokratisch verfassten Weltregierung. Globale Demokratie setzt auf basisdemokratische oder gemeinschaftsorientierte Prinzipien von in bestimmten Themenbereichen aktiven inter- oder transnationalen Organisationen nach dem Vorbild sozialer Bewegungen. Kosmopolitische Demokratie beabsichtigt »einen transnationalen Ausbau der allgemeinen Struktur politischen Handelns [...], der alle Ebenen und alle Mitwirkenden beim globalen Regieren umfasst, von Staaten, multinationalen Konzernen und internationa- 
len Institutionen bis hin zu sozialen Bewegungen und einzelnen Individuen« (ebd.: 122); sie zielt damit auf einen globalisierten Föderalismus gemäß dem Prinzip eines Bundesstaats oder Staatenbunds $a b$ - ein föderalistisch aufgebautes Europa könnte hier als Muster dienen.

(e) Ernesto Laclau und Chantal Mouffe begreifen diese Fundamentalisierung als Radikalisierung und Pluralisierung zugleich und nehmen sich des »Projekts für radikale Demokratie« (1991: 221) an. Es ist gekennzeichnet durch »die Ablehnung von privilegierten Bruchpunkten und der Vorstellung des Zusammenfließens der Kämpfe zu einem einheitlichen politischen Raum sowie im Gegensatz dazu die Anerkennung der Pluralität und Unbestimmtheit des Sozialen « (ebd.: 192). Mit dem Eigenschaftswort >politisch/ spielen Laclau und Mouffe nicht auf Parteien und Staat an, sondern auf einen

»Handlungstyp, dessen Ziel die Transformation eines sozialen Verhältnisses ist, das ein Subjekt in einem Verhältnis der Unterordnung konstruiert. [...] Was wir hervorheben wollen ist, dass Politik als eine Praxis des Erzeugens, der Reproduktion und Transformation sozialer Verhältnisse nicht auf einer bestimmten Ebene des Gesellschaftlichen verortet werden kann, da das Problem des Politischen das Problem der Einrichtung des Sozialen ist, d.h. der Definition und Artikulation sozialer Beziehungen auf einem kreuz und quer von Antagonismen durchzogenen Feld.« (1991: 193)

Für die >demokratische Revolution « »musste sich zuerst das demokratische Prinzip der Freiheit und Gleichheit als neue Matrix des sozialen Imaginären durchsetzen bzw., in unserer Terminologie, einen fundamentalen Knotenpunkt in der Konstitution des Politischen bilden « (ebd.: 195). Laclau und Mouffe erkennen für die heutige Zeit »eine neue Ausdehnung egalitärer Äquivalenzen und dadurch eine Ausweitung der demokratischen Revolution in neue Richtungen « (ebd.: 199), mit anderen Worten: »eine Ausdehnung der demokratischen Revolution auf eine Reihe ganz neuer sozialer Verhältnisse« (ebd.: 201), die insbesondere durch die »neuen sozialen Bewegungen « vorangetrieben wird. Dieser Prozess vollzieht sich allerdings als ein Wechselspiel von Identität und Differenz, Kontinuität und Diskontinuität. Zum einen nämlich stellen sich solche gesellschaftlichen Kämpfe um der besseren Durchschlagskraft willen in die hegemoniale Tradition der Demokratisierung, zum anderen aber handelt es sich um unverbundene, je besondere Auseinandersetzungen, denn »alle Kämpfe $[\ldots]$ haben $[\ldots]$ einen partiellen Charakter und können mit ganz unterschiedlichen Diskursen artikuliert werden« (ebd.: 211). Laclau und Mouffe fokussieren unter den demokratischen Grundwerten auf Freiheit und Gleichheit: »Insoweit von den beiden großen Themen des demokratischen Imaginären - Gleichheit und Freiheit die Gleichheit traditionell vorherrschte, verleihen nun die Forderungen nach Autonomie der Freiheit eine immer zentralere Rolle. Aus diesem Grund manifestieren sich viele dieser Widerstandsformen nicht in kollektiven Kämpfen, sondern über einen zunehmenden Individualismus.« (ebd.: 206) 
Wie Negt datieren auch Laclau und Mouffe und finden, »dass das demokratische Imaginäre seit den sechziger Jahren eine fundamentale Rolle für den Ausbruch neuer Forderungen gespielt hat« (ebd.: 207). Unter ihrem der Geschichte zugewandten Blick wird Demokratie radikal und plural: »In einem grundlegenden Sinne ist das Projekt einer radikalen und pluralen Demokratie deshalb nichts anderes als der Kampf um ein Höchstmaß an Autonomisierung von Bereichen auf der Basis der Verallgemeinerung der äquivalentiell-egalitären Logik.« (ebd.: 209) »Radikal und plural« insofern, als jedes Glied in der Kette demokratischer Kämpfe »in sich selbst das Prinzip seiner Geltung findet« (ebd.), und »demokratisch« insofern, als sich diese Selbstkonstitution aus der Verschiebung des »egalitären Imaginären« ergibt - Differenz und Identität, Diskontinuität und Kontinuität, wie gesagt. Laclau und Mouffe erkennen durchaus die hier angelegte »Unvereinbarkeit zwischen der Vermehrung politischer Räume, die einer radikalen Demokratie angemessen sind, und der Konstruktion kollektiver Identitäten auf der Basis der Äquivalenzlogik« (ebd.: 225). Sie behelfen sich angesichts dieser »offensichtlichen Dichotomie/Autonomie/Hegemonie« (ebd.) mit Wittgensteins Begriff der »Familienähnlichkeiten« (ebd.: 223) aus dessen Philosophischen Untersuchungen (vgl. 67. Abschnitt), um auf die grundlegende Verwandtschaft hinzuweisen. Keinen Zweifel lassen sie daran aufkommen, dass der demokratische Wandel unserer Zeit fundamentale Differenzierungen mit sich bringt: »Wir erleben eine Politisierung, die viel radikaler als jede uns bisher bekannte ist, weil sie dazu tendiert, die Unterscheidung zwischen dem Öffentlichen und dem Privaten aufzulösen - nicht im Sinne des Eingriffs in das Private durch einen einheitlichen öffentlichen Raum, sondern im Sinne einer Vermehrung radikal neuer und verschiedener politischer Räume.« (ebd.: 225)

Schließlich zögern sie in Anbetracht neuer Hierarchisierungstendenzen nicht, eine politische Empfehlung zu formulieren: »Angesichts des Projekts zur Rekonstruktion einer hierarchischen Gesellschaft sollte die Alternative der Linken darin bestehen, sich selbst vollständig auf dem Feld der demokratischen Revolution zu verorten und die Äquivalenzketten zwischen den verschiedenen Kämpfen gegen Unterdrückung zu erweitern.« (ebd.: 219) Darum wollen sie nicht auf die »liberal-demokratische Ideologie« verzichten, sondern sie in Richtung radikaler und pluraler Demokratie ausweiten. Dabei warnen sie vor jeglichem »essentialistischen Apriorismus« (ebd.: 220), sei es Klassismus, Etatismus, Ökonomismus, oder was auch immer.

Der »Respekt gegenüber den Gleichheitsrechten anderer untergeordneter Gruppen« (ebd.: 228), aber auch das Eigeninteresse an der »Verstärkung bestimmter demokratischer Kämpfe erfordert die Ausdehnung von Äquivalenzketten hin zu anderen Kämpfen« (ebd.: 226). Laclau und Mouffe erwähnen als Beispiel, dass Antirassismus, Antisexismus und Antikapitalismus aufeinander angewiesen seien. Da solche Äquivalenz jedoch stets unsicher ist, ergänzen Laclau und Mouffe deren Logik durch die »Logik der Autonomie«: 
»Aus eben diesem Grund reicht die Forderung nach Gleichheit nicht aus, sondern muss durch die Forderung nach Freiheit ausgeglichen werden, damit wir von einer radikalen und pluralen Demokratie sprechen können. Eine radikale und nicht-plurale Demokratie würde einen einzigen Raum von Gleichheit auf der Basis unbegrenzter Wirksamkeit der Äquivalenzlogik konstituieren und das irreduzible Moment der Pluralität von Räumen nicht anerkennen. Dieses Prinzip der Teilung von Räumen ist aber die Basis der Forderung nach Freiheit.« (ebd.: 228)

Es ist zugleich die Nahtstelle zum Liberalismus. Man könnte sagen: Wirksame Orientierung an Gleichheit radikalisiert, wirksame Orientierung an Freiheit pluralisiert. Letztlich plädieren Laclau und Mouffe für eine »Polyphonie der Stimmen«, in der sie den Abschied vom Universellen für entscheidend halten:

»Es gibt keine radikale und plurale Demokratie ohne den Verzicht auf den Diskurs des Universalen und seiner impliziten Behauptung eines privilegierten Zugangspunktes zu >der Wahrheit $`$, die nur von einer begrenzten Zahl von Subjekten erreicht werden kann. [...] Rechtsinstitutionen, das Erziehungssystem, Arbeitsbeziehungen, die Diskurse des Widerstands marginaler Gruppen konstruieren eigenständige und irreduzible Formen sozialen Protestes und tragen dadurch alle zur diskursiven Komplexität und Reichhaltigkeit bei, auf die sich das Programm einer radikalen Demokratie stützen sollte.« (ebd.: 237)

Laclau und Mouffe fokussieren auf Gleichheit und Freiheit, wenngleich es alle Grundwerte im Auge zu behalten gilt, welche der Demokratisierung Orientierung bieten. Ein etwas breiterer Wertebezug scheint z.B. bei Nancy Fraser durch, wenn sie versucht, »die Theorie kultureller Gerechtigkeit mit der Theorie der Verteilungsgerechtigkeit zu verbinden« (2001: 17). Wie Laclau und Mouffe erkennt Fraser in der "postsozialistischen Situation« nach 1989 eine Vielzahl von Kämpfen, d.h., viele unterschiedliche soziale und kulturelle Auseinandersetzungen. Die analogen, scheinbaren Gegensätze sozialistisch vs. postsozialistisch, ökonomisch definierte Klassen vs. kulturell definierte Wertegemeinschaften, Gleichheit vs. Differenz, Umverteilung vs. Anerkennung, Sozialpolitik vs. Kulturpolitik, Klassenpolitik vs. Identitätspolitik entlarvt sie (ebd.: 11f.) allerdings als »falsche Antithesen«. Laclaus und Mouffes Frage nach Autonomie und Hegemonie kehrt bei Fraser wieder als ein »Umverteilungs-Anerkennungs-Dilemma«: »Menschen, die sowohl von kultureller Ungerechtigkeit als auch von ökonomischer Ungerechtigkeit betroffen sind, benötigen Anerkennung ebenso sehr wie Umverteilung. D.h., sie müssen einerseits ihre Besonderheit geltend machen und andererseits verleugnen« (ebd.: 33). Fraser ordnet politische Strategien in einem Vier-Felder-Schema und hält die »Kombination aus Sozialismus und Dekonstruktion« (ebd.: 64) für die erfolgversprechendste Vorgehensweise. 
Tabelle 1: Maßnahmen gegen ökonomische und kulturelle Ungleichheiten

\begin{tabular}{|c|l|l|}
\hline $\begin{array}{c}\text { Maßnahmen } \\
\text { Gerechtig- } \\
\text { keitsaspekt }\end{array}$ & \multicolumn{1}{|c|}{ Affirmation } & \multicolumn{1}{c|}{ Transformation } \\
\hline Umverteilung & $\begin{array}{l}\text { Liberaler Wohlfahrtsstaat: } \\
\text { Oberflächliche Neuzutei- } \\
\text { lungen vorhandener Güter } \\
\text { an existierende Gruppen; } \\
\text { unterstützt Gruppendiffe- } \\
\text { renzierung; kann Missach- } \\
\text { tung erzeugen }\end{array}$ & $\begin{array}{l}\text { Sozialismus: } \\
\text { Gründliche Umstrukturie- } \\
\text { rung der Produktionsver- } \\
\text { hältnisse; weicht Gruppen- } \\
\text { differenzierung auf; kann } \\
\text { manche Formen der Miss- } \\
\text { achtung abschaffen helfen }\end{array}$ \\
\hline Anerkennung & $\begin{array}{l}\text { Üblicher Multikulturalis- } \\
\text { mus: } \\
\text { Oberflächliche Neuzutei- } \\
\text { lung von Respekt an beste- } \\
\text { hende Identitäten existie- } \\
\text { render Gruppen; unterstützt } \\
\text { Gruppendifferenzierungen }\end{array}$ & $\begin{array}{l}\text { Dekonstruktion: } \\
\text { Gründliche Umstrukturie- } \\
\text { rung von Anerkennungs- } \\
\text { verhältnissen; destabilisiert } \\
\text { Gruppendifferenzierung }\end{array}$ \\
\hline
\end{tabular}

(Quelle: nach Fraser 2001: 55; vgl. auch Fraser 2002: 102ff.)

Nach unserem Durchgang durch wichtige zeitgenössische theoretische Positionen und empirische Einschätzungen erscheint die demokratische Revolution als ein offenes Projekt, das auch in unserer Zeit noch nicht zu seinem Abschluss gekommen ist. »Wenn Sie mich also fragen, ob die Demokratie eine Zukunft hat und gesetzt, sie habe eine, welcher Art diese Zukunft ist, so kann ich nur in aller Ruhe antworten: Ich weiß es nicht «, merkte Bobbio (1988: 8) einmal an. Führte die demokratische Revolution, beginnend im 18. Jahrhundert, zur Demokratie als politischer Institution, so die Erfassung vieler Institutionen weiterer Lebensbereiche durch den Geist des Demokratismus zur Demokratie als Lebensform. Die Demokratisierung der Demokratie schafft auch nötige Voraussetzungen für eine lebendige Demokratie. Bezugspunkt ist das >demokratische Imaginäre `, das immer wieder neu artikuliert wird. Die demokratische Revolution vollzieht sich nicht als einheitliches Projekt, gleichsam >aus einem Guss`, vielmehr realisiert sie sich durch eine Vielzahl einzelner Kämpfe. Zwei sich widersprechende Logiken sind zugleich am Werke: eine Logik der Vervielfältigung (Autonomie), die von zahlreichen einzelnen Negationen diskriminierender Verhältnisse lebt, und eine Logik der Vereinheitlichung (Hegemonie). Radikal wird Demokratie, wenn Mitbestimmung und mitbestimmungsförderliche Voraussetzungen sich in tendenziell allen Lebensbereichen ausdehnen. Plural wird Demokratie, wenn sich die Schauplätze dieses Geschehens nicht bloß vervielfältigen, sondern (auch) eigensinnig entwickeln. »Demokratie ist nicht, sondern wird ständig« (Beyme 1994: 9) - und der Tendenz nach überall.

Die vielfältigen Kämpfe, die im Geiste des Demokratismus geführt werden, werden nicht mehr nur traditionell, im Zusammenspiel von Medienöf- 
fentlichkeit, Politik, Recht und Verwaltung, ausgetragen. Mediatisierung, Politisierung, Verrechtlichung, Bürokratisierung - was öffentlich diskutiert sowie politisch debattiert und entschieden wird, wird rechtlich durchgesetzt und administrativ umgesetzt - bleiben nach wie vor für Demokratien lebensnotwendige demokratisierende Prozesse. Aber die Rationalisierung als »kommunikative Verflüssigungtraditionsfester [...] Institutionen« (Habermas 1981: 139), als »kommunikative Verflüssigung von traditionalen Pflichten und Loyalitäten« (Habermas 2004: 77) modernisiert tendenziell sämtliche Lebensbereiche und unterstützt in ihnen Demokratiediskurs wie Demokratiepraxis, und dies im Kleinen wie im Großen.

Traut man demokratischen Werten theoretisch und praktisch zu, dass sie im menschlichen Zusammenleben verwirklicht werden, so müsste sich deren Wirklichkeit, Verwirklichung und Verwirklichtheit in Beschreibungen von Gesellschaft wiederfinden lassen. Im Folgenden sei ein theoretischer Rahmen, ein Gesellschaftsbild entworfen, das diese Verortung ermöglicht.

\section{Das GesellschaftSBiLd DER MODALEN STRUKTURIERUNGSTHEORIE}

Die Modale Strukturierungstheorie (Husi 2010) beerbt insbesondere zwei Theorietraditionen. Von der Tradition der Praxistheorien (Reckwitz 2003), in der u.a. Pierre Bourdieu $(1979,1987)$ und Anthony Giddens $(1984,1988)$ stehen, bezieht sie den Ausgangspunkt aller weiteren Überlegungen: die menschliche Praxis. Und von Jürgen Habermas' Theorie des kommunikativen Handelns lässt sie sich dazu anregen, von Sprachanalyse auszugehen, um letztlich eine Gesellschaftstheorie zu entwickeln. Die Bezeichnung der im Weiteren zu beschreibenden Theorie enthält zwei Teile: >modal und 〉Strukturierung〈, die die beiden genannten Erbschaften widerspiegeln.

Uwe Schimank bezeichnet als »Gegenstand der Soziologie die fortlaufende wechselseitige Konstitution von sozialem Handeln und sozialen Strukturen« (2000: 9). Präzisierend hält Hans-Peter Müller als »Grundfragen einer jeglichen Sozialtheorie« die folgenden fest: »Was heißt Handeln? Was meint Struktur? Wie sind Handeln und Struktur relationiert?« (2005: 21). Während in der Soziologie tatsächlich weitgehend Konsens darüber besteht, dass ihr Gegenstand Gesellschaftsstruktur und gesellschaftliche Praxis darstellen, so gehen die Meinungen auseinander, wie denn Struktur einerseits und Praxis andererseits sowie ihr Verhältnis zueinander, die Strukturierung, genau aufzufassen sind. Der führende englische Soziologe, Anthony Giddens, hat hierzu seine >Theorie der Strukturierung « entworfen und mit dieser Namensgebung gleich auch ausgedrückt, dass Strukturierung besondere theoretische Aufmerksamkeit verdient. Zentral ist der Gedanke, dass Struktur und Praxis keine einander bloß gegenübergestellte, voneinander weitgehend unabhängige Pole bezeichnen - Giddens distanziert sich gleichermaßen von 
Theorieangeboten mit >objektivistischer` wie >subjektivistischer Schlagseite, die entweder Struktur oder Praxis verabsolutieren. Vielmehr dient Struktur der Praxis medial, und Praxis reproduziert Struktur und kann diese zuweilen auch tiefgreifend verändern. Giddens (1988: 77ff.) nennt diesen Gedanken, dass Struktur sowohl Medium als auch Folge von Praxis ist, »Dualität von Struktur«. Er fasst Struktur als Regeln und Ressourcen auf. Zwischen Struktur und Praxis vermitteln »Strukturierungsmodalitäten «, und mit diesem Begriff gelangt man zum zweiten Teil der Theoriebezeichnung >Modale Strukturierungstheorie` und zugleich zu Habermas' Idee einer sprachwissenschaftlichen Grundlegung. Zentraler Gedanke der Modalen Strukturierungstheorie ist es nämlich, dass sich Strukturierungsmodalitäten - dasjenige also, das zwischen Struktur und Praxis vermittelt - durch nichts besser verstehen und wiedergeben lassen als durch Modalverben: Unsere Alltagssprache selber stellt uns die begrifflichen Mittel zur Verfügung, mit der die Vermittlung von Struktur und Praxis tatsächlich verstanden werden kann. Gleichzeitig lassen Modalverben präzise die grundlegenden Differenzierungen von Struktur und Praxis erscheinen. Damit kann das von Giddens so bezeichnete Problem der »doppelten Hermeneutik« elegant angegangen werden, das die Frage aufwirft, wie sich sozialwissenschaftliche Beobachtungen zweiter Ordnung auf alltägliche Beobachtungen erster Ordnung beziehen. Modalverben zählen, wie die Linguistik empirisch zu belegen weiß, zu den am häufigsten verwendeten sprachlichen Mitteln. Zugleich verweisen genau sie auf die wichtigsten, sozialwissenschaftlich zu erfassenden Differenzierungen gesellschaftlicher Struktur und Praxis und lassen letztere beide zueinander in Beziehung setzen.

Linguistische Versuche zeigen, dass sich Modalverben auf unterschiedliche Art und Weise ordnen und verstehen lassen. Am überzeugendsten fällt jener Versuch aus, der die Modalverben auf menschliches Innenleben, Zusammenleben sowie Dinge bezieht, anders gesagt: auf die subjektive, soziale und objektive Welt. Bei diesem Bezug nämlich bilden sich drei Paare von Modalverben aufgrund dessen, dass zwei davon spezifische Weltbezüge aufweisen und ein Paar nicht: Mögen und wollen wurzeln in der einzelnen Individuen privilegiert zugänglichen subjektiven Welt, dürfen und sollen in der gemeinsam zugänglichen sozialen Welt - soweit die spezifischen Weltbezüge; können und müssen dagegen wurzeln in allen drei Bezugswelten, d.h. mithin, nicht nur in der objektiven Welt.

Es sei nur nebenbei bemerkt und nicht näher ausgeführt, dass sich die paarweise Anordnung der Modalverben auch in den beiden genannten Praxistheorien erkennen lässt: Bei Giddens stehen dafür Regeln und Ressourcen als Strukturbestandteile sowie sein »Stratifikationsmodell der Handelnden«, das sich auf Kognition und Motivation bezieht; bei Bourdieu seine zentralen theoretischen Konzepte Kapital, Habitus und Feld.

Mit den Modalverben lässt sich noch eine weitere zentrale Einsicht Giddens' verknüpfen, die jeweils die Differenz zwischen den beiden zu Paaren zusammengefassten Modalverben erhellt: Struktur schränkt Praxis nicht nur 
ein - so eine gängige Vorstellung -, sondern ermöglicht sie auch. Ermöglichungen öffnen, Einschränkungen schließen Handlungsspielräume (und Erlebensspielräume). Beide sind, um es zu wiederholen, in die Praxis eingewoben und werden durch sie auch hervorgebracht, sei es bestätigt oder verändert. Gleich auf den ersten Blick lässt sich erkennen, dass können und dürfen Ermöglichung repräsentieren, müssen und sollen dagegen Einschränkung. Diese Modallogik zeigt sich auch darin, dass müssen >nicht anders können` bedeutet, und analog dazu lässt sich sollen als >nicht anders dürfen begreifen. Bei mögen und wollen liegt der Fall ein wenig komplizierter, jedoch auch hier: Mögen eröffnet einem Menschen zunächst motivational Möglichkeiten, deren Vielfalt er durch sein entschiedenes Wollen wieder selber minimiert.

\section{Tabelle 2: Strukturierung und Differenzierung in der Modalen}

\section{Strukturierungstheorie}

\begin{tabular}{|c|c|c|c|c|}
\hline $\begin{array}{l}\text { Modal- } \\
\text { verben }\end{array}$ & Medium & $\begin{array}{c}\text { Bezug auf } \\
\text { Welt }\end{array}$ & $\begin{array}{c}\text { Strukturie- } \\
\text { rungs- } \\
\text { modalität }\end{array}$ & $\begin{array}{l}\text { Differenzie- } \\
\quad \text { rung }\end{array}$ \\
\hline können & ermöglichend & \multirow{2}{*}{$\begin{array}{c}\text { subjektive } \\
\text { soziale } \\
\text { objektive }\end{array}$} & \multirow{2}{*}{ instrumentale } & \multirow{2}{*}{ hierarchische } \\
\hline müssen & einschränkend & & & \\
\hline mögen & ermöglichend & \multirow{2}{*}{ subjektive } & \multirow{2}{*}{ motivationale } & \multirow{2}{*}{ kulturelle } \\
\hline wollen & einschränkend & & & \\
\hline dürfen & ermöglichend & \multirow{2}{*}{ soziale } & \multirow{2}{*}{ regulative } & \multirow{2}{*}{ institutionelle } \\
\hline sollen & einschränkend & & & \\
\hline
\end{tabular}

Indem man also Weltbezüge und Mediumsqualitäten kombiniert, erhält man ein vollständiges Tableau der Modalverben und somit der Strukturierungsmodalitäten. Giddens' Theorem der Dualität von Struktur lässt sich demnach so interpretieren, dass Können, Dürfen und Mögen ermöglichend und Müssen, Sollen und Wollen einschränkend unablässig strukturierend in die Praxis von Menschen medial einfließen. Die Praxis ihrerseits reproduziert diese strukturellen Voraussetzungen und verstetigt sie auf diese Weise, wobei Veränderungen im Kleinen und Großen stets möglich sind. Diese Verstetigungen lassen sich mit einer lebenssoziologischen Begrifflichkeit ausdrücken, welche die relativ dauerhaften Teile der individuellen Lebensstruktur wiedergibt: Lebenslage steht für die Gesamtheit der Mittel und Zwänge, Lebensziele für die Gesamtheit der Wünsche und Ziele, Rollen für die Gesamtheit der Rechte und Pflichten eines Menschen. Welche Lebenslagen, Lebensziele und Rollen ein Mensch erwirbt und erhält, ist insbesondere durch Geschlecht, Alter und Ethnie beeinflusst. Aus der Denktradition des Strukturalismus stammt die Erkenntnis, dass einzelne Strukturelemente, hier also die konkreten, noch näher zu bestimmenden Bestandteile individueller 
Lebenslagen, Lebensziele und Rollen, ihre Bedeutung erst im Kontext aller anderen Elemente erhalten. Das bedeutet, der Vergleich mit anderen Menschen erst legt diese Bedeutungen fest. Das führt zur Betrachtung individueller Lebensstruktur im Kontext der Gesellschaftsstruktur. Der Vergleich klärt ferner empirisch darüber auf, inwiefern sich in einer gegebenen Gesellschaft große Gruppierungen von Menschen mit ähnlichen Lebenslagen und/oder Lebenszielen und/oder Rollen finden. Für diese Gruppierungen stehen zunächst die Begriffe Klasse und Milieu, während ein Lebensbereich Menschen versammelt, die darin aufeinander verweisende Leistungs- und Empfangsrollen spielen, die sich inhaltlich deutlich von anderen Rollen in anderen Lebensbereichen unterscheiden. Gesellschaften sind insofern hierarchisch, kulturell und institutionell differenziert, als sich solche Klassen, Milieus und Lebensbereiche empirisch finden lassen. Vor diesem Hintergrund der drei Differenzierungsweisen wird erkennbar, dass sich Menschen in unterschiedlichen Hinsichten begegnen können, nämlich als einander überoder untergeordnet; als miteinander vertraut oder einander fremd; und als solche, die denselben oder anderen Regeln unterworfen sind.

\section{Tabelle 3: Individuelle und gesellschaftliche Struktur in der Modalen} Strukturierungstheorie

\begin{tabular}{|l|l|l|l|}
\hline $\begin{array}{l}\text { Modal- } \\
\text { verben }\end{array}$ & $\begin{array}{l}\text { individuelle } \\
\text { Lebensstruktur }\end{array}$ & $\begin{array}{l}\text { Gesellschafts- } \\
\text { struktur }\end{array}$ & $\begin{array}{l}\text { Verhältnisse zwischen } \\
\text { Menschen }\end{array}$ \\
\hline $\begin{array}{l}\text { können \& } \\
\text { müssen }\end{array}$ & Lebenslage & Klassen & Über- und Unterordnung \\
\hline $\begin{array}{l}\text { mögen \& } \\
\text { wollen }\end{array}$ & Lebensziele & Milieus & $\begin{array}{l}\text { Vertrautheit und Fremd- } \\
\text { heit }\end{array}$ \\
\hline $\begin{array}{l}\text { dürfen \& } \\
\text { sollen }\end{array}$ & Rollen & Lebensbereiche & $\begin{array}{l}\text { gleiche bzw. unterschied- } \\
\text { liche Geregeltheit }\end{array}$ \\
\hline
\end{tabular}

Giddens schreibt der bis dahin beschriebenen Struktur eine >virtuelle` Existenz zu. Praxis vollzieht sich dagegen konkret situativ in Raum und Zeit als ein Fluss des Handelns und Erlebens, der sich eingelebter Praktiken bedient. Praxis erweckt Struktur gleichsam zum Leben. Wie auf der Seite der Struktur kommt es zu Verstetigungen, d.h. zu Routinen, Gewohnheiten. Praxistheorien akzentuieren diesen Aspekt der Praxis, ohne zu leugnen, dass immer auch Überraschungen möglich sind. Zwischen Struktur und Praxis besteht demnach keine deterministische Beziehung, sondern eine Beziehung der Wahrscheinlichkeit, und zugleich wird auf diese Weise ersichtlich, dass Struktur und Praxis dazu tendieren, sich wechselseitig zu verstetigen.

Auch hinsichtlich der Praxis kommt eine lebenssoziologische Begrifflichkeit zum Zuge: Individuelle Lebenspraxis verdichtet sich im Alltag zu einer Lebensweise als der Gesamtheit der Handlungen eines Menschen, die mit einem Lebensgefühl, als einer relativ dauerhaften Einschätzung des eigenen Lebens, einher geht. Man kann vermuten, dass ein gutes Lebensge- 
fühl dann entsteht, wenn es gelingt, Lebenslage, Lebensziele, Rollen und Lebensweise aufeinander abzustimmen.

\section{Abbildung 2: Struktur und Praxis in der Modalen Strukturierungstheorie}



Der Routinecharakter der Alltagspraxis beinhaltet, dass Menschen ihre Praxis als einen Fluss des Tuns und Lassens leben. Es handelt sich dabei, so Giddens, mehr um ein Handeln als um Handlungen. Handlungen, als klar voneinander abgegrenzte, bilden eher die Ausnahme, sie treten hervor, wenn Störungen des Handlungsflusses aufmerken lassen, die Aufmerksamkeit wecken. Dem Handlungsfluss entspricht ein innerer Strom des Erlebens, d.h. des Wahrnehmens, Denkens, Fühlens.

Menschen erleben sich in ihrem Alltag wechselseitig als Handelnde, als Individuen also, die sich nicht auf eine völlig beliebige, zufällige Art oder im Gegenteil auf eine völlig determinierte Weise verhalten, sondern ihre Körperbewegungen und Sprechhandlungen wählen können und mit ihrer jeweiligen Wahl einen Sinn verbinden. Handeln geschieht demnach zwar wissentlich und willentlich, Praxistheorien bewahren jedoch davor, diese beiden Momente des Handelns zu überbetonen. Das Gewohnte minimiert den Energieaufwand der Praxis für Sinn und Wille.

In Interaktionen stabilisieren Menschen wechselseitig ihre Lebenspraxen und schauen Praktiken voneinander ab. Missverständnisse und Konflikte bilden die Ausnahme. So gleichen sich bei ähnlichen strukturellen Gegebenheiten auch die Lebenspraxen. Entsprechend der von den Modalverben 
hergeleiteten drei Differenzierungsarten bilden sich besondere Klassenpraxen, Milieupraxen und Lebensbereichspraxen aus. Mit anderen Worten: Angehörige einer bestimmten Klasse, eines bestimmten Milieus oder Lebensbereichs handeln und erleben auf je ähnliche Art und Weise.

Das >Gesellschaftliche` der Praxis tritt indessen erst vollends zutage, wenn nicht nur solche Ähnlichkeiten festzustellen sind, sondern Ein- und Ausschlüsse in Betracht kommen. Die Differenzierung der Gesellschaftsstruktur widerspiegelt sich in der praktischen Differenzierung, die sichtbar wird, wenn sich Subsysteme bilden, die Grenzen aufweisen. Klassen, Milieus und Lebensbereiche, nun verstanden als Handlungszusammenhänge, schließen zugleich ein und aus. Menschen erleben in ihrem Alltag dauernd Inklusion in Handlungszusammenhänge und Exklusion aus ihnen, seien es Klassen, Milieus oder Lebensbereiche - man wird nicht überall zugelassen, man will auch nicht überall zugelassen werden, man bleibt aber oft unfreiwillig außen vor. Kurzum, Klassen-, Milieu- und Lebensbereichsangehörige, anders gesagt Gleichgestellte, Gleichgesinnte und Gleichgeregelte, neigen dazu, unter ihresgleichen zu bleiben. Was für die einen Eingrenzung bedeutet, bedeutet für die anderen Ausgrenzung. Menschen schauen nicht nur voneinander $a b$, sondern sehen auch voneinander $a b$. Alle individuelle und gemeinsame Praxis, jegliches Handeln und Erleben findet in Raum und Zeit statt, zu einem bestimmten Zeitpunkt an einem bestimmten Ort, im Hier und Jetzt eines bestimmten handelnden und erlebenden Menschen.

Wie können nun die sechs demokratischen Grundwerte, Freiheit, Gleichheit, Sicherheit zunächst und sodann Toleranz, Solidarität, Frieden, im skizzierten Gesellschaftsbild der Modalen Strukturierungstheorie zur Darstellung gebracht werden?

\section{DIE SECHS DEMOKRATISCHEN GRUNDWERTE IM RAHMEN DER MODALEN STRUKTURIERUNGSTHEORIE}

Nicht nur über den Kanon der Grundwerte wird kontrovers diskutiert, sondern ebenso über die zutreffende Interpretation jedes Grundwerts. An dieser Stelle muss eine angemessene Darstellung nur schon der wichtigsten Wertinterpretationen ausbleiben, sie ist auch nicht das Ziel. Freilich lassen sich konsensfähige Bedeutungskerne herausschälen und dabei helfen zunächst Giddens' Unterscheidung von Struktur und Handeln und sein differenzierendes Verständnis von Strukturierung als Ermöglichung und Einschränkung weiter. Die Modale Strukturierungstheorie lässt anhand der Modalverben die Weltbezüge der Grundwerte sodann noch präziser erfassen.

Freiheit (Husi/Meier Kressig 1998: 360ff., 371ff.) wird als negative und positive Freiheit interpretiert: Die >Freiheit von $<$ geht einher mit der >Freiheit zu<. Negative Freiheit ist die Freiheit von Einschränkungen, positive Freiheit ist die Freiheit durch Ermöglichungen. Dies kann noch nach Moda- 
litäten, insbesondere hierarchischer und institutioneller Modalität, aufgeschlüsselt werden: Negative Freiheit bezieht sich auf das Freisein von Zwängen und Pflichten, positive Freiheit auf das Vorhandensein von Mitteln und Rechten. In Bezug auf die motivationale Modalität, Wünsche und Ziele also, verhält es sich nicht ganz so einfach. Dem Wollen voran geht, was der Wille überhaupt vermag: sogenannte volitive Fähigkeiten (die noch der instrumentellen Modalität zugehören). Hier ist Willensfreiheit bedeutsam. Sie bezieht sich auf das Vermögen, eine Handlung und zugleich eine andere Handlung zu wollen (und nicht: zu mögen oder zu tun). Ein völlig unfreier Wille kann dagegen nicht anders wollen, muss so wollen, d.h. unterliegt einem Zwang. »Willensfreiheit ist in nuce ein Anders-Wollen-Können in Bezug auf Handlungen sowie den eigenen Charakter, wogegen sich Handlungsfreiheit in der Durchsetzung des eigenen Willens gegen innere und äußere Hindernisse manifestiert« (Husi/Meier Kressig 1998: 397). Mit Blick auf die motivationale Modalität sodann kann die Unterscheidung positiv/negativ folgendermaßen interpretiert werden: Wünsche wirken ermöglichend und verkörpern positive Freiheiten insofern, als sie Handelnden mögliche Beweggründe, die in ihren Werten gründen, vor Augen führen. Ziele wirken einschränkend und verkörpern, wenn man so will, negative Unfreiheiten insofern, als sie Handelnde auf eine Handlung festlegen.

Gleichheit ist die Abwesenheit dessen, »dass einige Menschen gegenüber anderen nicht einfach als in bestimmter Hinsicht verschieden erscheinen, sondern dadurch gleichzeitig auch als besser- oder schlechter-, höheroder tiefer gestellt, bevorrechtigt oder benachteiligt« (Bolte/Hradil 1988, zit. nach Husi/Meier Kressig 1998: 206). Gleichheit zeigt sich also in Form gleicher Mittel und Zwänge sowie gleicher Rechte und Pflichten. Man könnte hier analog zu Freiheit von negativer Gleichheit in Bezug auf Zwänge und Pflichten und positiver Gleichheit in Bezug auf Mittel und Rechte sprechen.

Von Gleichheit zu Gerechtigkeit führt kein weiter Weg. In der Gerechtigkeit sind Freiheit und Gleichheit vereint, denn »Gerechtigkeit bezieht sich auf die gleichen Freiheiten unvertretbarer und sich selbst bestimmender Individuen« (Habermas 1991: 70). Bereits Marx und Engels haben denn im 1848 veröffentlichten Manifest der Kommunistischen Partei eine künftige Gesellschaft vor Augen, "worin die freie Entwicklung eines jeden die Bedingung für die freie Entwicklung aller ist.« (1959: 482).

Freiheit, Gleichheit und Gerechtigkeit sind also Grundwerte, die sich auf Struktur beziehen, die über Strukturierung der Praxis als Medium dient. Auf dieselbe Weise lässt sich auch Sicherheit interpretieren (Husi/Meier Kressig 1998: 281ff.). Sicherheit ist dauerhaftes Geschütztsein, nämlich vor Einschränkungen und von Ermöglichungen: Erwünschtes besteht und hat Bestand, Unerwünschtes droht nicht. Dauerhaftigkeit, Beständigkeit zeitigt Erwartbarkeit. Sicherheit erweist sich also ebenso in erwartbarer Abwesenheit von Einschränkungen wie in erwartbaren Ermöglichungen. Hierbei lässt sich die bereits bei Freiheit hilfreiche Unterscheidung wieder aufgreifen: 
Negative Sicherheit ist demnach beständiger und darum erwartbarer Schutz vor Zwängen und Pflichten, und positive Sicherheit ist beständiger und darum erwartbarer Schutz von Mitteln und Rechten. Risiken und Gefahren lauern, wo Einschränkungen drohen und Ermöglichungen womöglich zu wenig geschützt sind. Eine Beziehung zwischen Freiheit und Sicherheit besteht insofern, als negative Sicherheit der Erwartbarkeit negativer Freiheit entspricht und positive Sicherheit der Erwartbarkeit positiver Freiheit. Der Blickwinkel der Sicherheit bereichert denn auch das Verständnis von Gerechtigkeit: Gerechtigkeit bedeutet schließlich sichere gleiche Freiheiten. Es kommt nicht von ungefähr, dass zum Rechtsstaat wesentlich auch Rechtsgleichheit und Rechtssicherheit gehören, während Rechte an sich Freiheiten verkörpern. Gerechtigkeit lässt sich demnach mit Bezug auf Gesellschaftsstruktur begreifen als gleich sichere gleiche negative Freiheit von Einschränkung und positive Freiheit der Ermöglichung.

Freiheit, Gleichheit, Sicherheit wie auch Gerechtigkeit beziehen sich auf erwünschte Qualitäten der Strukturierung von Praxis. Praxis erscheint im Lichte dieser primären Grundwerte als auf bestimmte, befriedigende oder unbefriedigende Weise ermöglicht und eingeschränkt. Drei weitere Grundwerte, die sekundären, beziehen sich auf Eigenschaften gelebter sozialer Beziehungen: Tolerantes, solidarisches, friedliches Handeln zeigt sich im Bezug auf andere Menschen - ohne Mitmensch keine Toleranz, keine Solidarität, kein Friede. Ein Individuum, das in seinem Handeln diese drei Grundwerte verwirklicht, richtet sich auf ein anderes Individuum, indem es dessen Handeln toleriert, sich mit ihm solidarisiert und sich ihm gegenüber friedlich zeigt. Abwertung, verweigerte mögliche Hilfe, Aggression stehen dem gegenüber. Menschliches Handeln befindet sich also im Spannungsfeld von anerkennen und abwerten, unterstützen und im Stich lassen, sich zurückhalten und verletzen. In den Wahlen der Handelnden in diesen Spannungsfeldern verwirklichen sich Toleranz, Solidarität und Frieden.

Toleranz erweist sich im Verzicht auf negative Sanktion eines Handelns, das von einer geltenden Norm abweicht. Tolerantes Handeln ist norm- und abweichungsbewusstes, sanktionsloses Handeln gegenüber anderen. Es erstreckt sich von bloßer Duldung bis ausdrücklicher Förderung. Forst (2003: 42-48) findet denn in der Geschichte der Toleranz vier grundlegende Konzeptionen: die Erlaubnis-, Koexistenz-, Respekt- und WertschätzungsKonzeption. In der Toleranz zeigt sich »der grundlegende Respekt für andere als moralisch autonome Personen« (Forst 2003: 21). Im Spannungsfeld eigener und fremder Lebensziele und Rollen, genauer: ethischer Werte und moralischer Normen

»ergibt sich die für die Frage der Toleranz konstitutive Differenzierung zwischen (1) den eigenen ethischen Konzeptionen des Guten, die man vollständig bejaht, (2) allgemein geltenden moralischen Normen, (3) anderen Konzeptionen des Guten, die man kritisiert oder ablehnt, aber tolerieren kann (und muss), weil sie nicht unmoralisch sind, und (4) solchen Auffassungen, die man nicht primär mit ethischen, son- 
dern mit moralischen Gründen verurteilt, weil sie die Kriterien von Reziprozität und Allgemeinheit verletzen.« (Forst 2003: 21)

In diesem Spannungsfeld bietet daher die Unterscheidung von Moral und Ethos Orientierung.

»Sich und die Welt mit toleranten Augen zu sehen heißt, zwischen dem unterscheiden können, was Menschen moralisch voneinander fordern können, und dem, was für sie vielleicht viel bedeutsamer ist, nämlich den Auffassungen davon, was ein Leben lebenswert und gut macht. Und es heißt zu sehen, dass bezüglich Letzterem unendlicher Streit besteht, der aber weder die Geltung der Moral noch die Wahrheit der eigenen Überzeugungen oder die Integration einer Gesellschaft in Frage stellen muss.« (ebd.: 23)

Solidarität erweist sich in der Sorge um das Wohl der Mitmenschen, in der ausgleichenden Hilfe an Hilfsbedürftige. Will man Solidarität von Wohltätigkeit unterscheiden, so ist solidarisches Handeln mehr um gerechten Ausgleich bemüht. Es ist ausgleichendes Handeln und nicht nur ein einfaches Geben. Eine umfassende Definition stammt von Wildt (1998: 212f.):

') Solidarität bezeichnet eine engagierte Handlung oder Handlungsbereitschaft eines Akteurs gegenüber einem Rezipienten genau dann, wenn gilt

I. Bezüglich der unmittelbaren Intentionen des Akteurs:

(1) Akteur und Rezipient sind durch Gefühle der Zusammengehörigkeit oder Mitgefühl miteinander verbunden.

(2) Die Motivation des Akteurs ist mindestens teilweise altruistisch.

(3) Der Akteur versteht seine Handlung als Hilfe in einer Art Notlage des Rezipienten.

(4) Diese Notlage wird vom Akteur als moralisches Problem verstanden, und zwar als Ursprung einer Verpflichtung (meist auch als Unrecht an dem Rezipienten).

(5) Der Akteur glaubt, dass er selbst moralisch verpflichtet ist, entsprechend zu handeln.

(6) Der Akteur glaubt nicht, dass der Rezipient seiner Hilfe ein - juridisches oder auch nur moralisches - Recht auf diese hat.

II. Bezüglich der Annahmen des Akteurs über Intentionen des Rezipienten:

(7) Der Akteur unterstellt, dass der Rezipient seine Notlage ähnlich beurteilt wie er selbst.

(8) Der Akteur unterstellt, dass der Rezipient motiviert ist und, soweit möglich, ernsthaft versucht, seine Notlage zu bekämpfen.

(9) Der Akteur unterstellt mindestens die Möglichkeit, dass es analoge Situationen gibt, in denen der Rezipient sich (aus ähnlicher Motivation) ihm oder Dritten gegenüber analog verhält, verhalten hat oder verhalten wird«.

Die meisten Begriffsauffassungen sind weniger anspruchsvoll; kontrovers diskutiert wird, ob es um Hilfe unter Gleichen oder Ungleichen geht: »Soli- 
darität soll hier ein gemeinsames soziales Handeln bedeuten, bei dem eine Vielzahl von Menschen aus einer ihnen gleichen und gemeinsamen Lebenslage heraus und um gemeinsamer und gleicher Ziele willen, einem >sozialen Gegenpart` gegenüber füreinander einsteht« (Reitzenstein 1961, zit. nach Zoll 2000: 13; Herv. G.H.). Oder Solidarität bedeutet »Menschen, deren Lage man nicht teilt, dennoch bei der Verwirklichung derselben Chancen, Rechte und Ziele zu unterstützen, die man selbst geniesst bzw. als wertvoll erachtet« (Wildt 1996, zit. nach Zoll 2000: 17; Herv. G.H.). Bemerkenswerterweise macht sich der Unterschied der beiden Definitionen am Kriterium der Lebenslage fest.

Frieden erweist sich im Verzicht auf Gewaltanwendung, im verträglichen Zusammenleben. Friedliches Handeln ist gewaltloses Handeln, genauer noch: beständiges und darum erwartbares gewaltfreies Handeln. Es handelt sich also um mehr als um einen bloß zufälligen oder situativen Gewaltverzicht. Dabei wird gehofft, »dass gerade in der Demokratie das Experiment der Institutionalisierung friedlichen Konfliktes dauerhaft gelingen könne« (Kielmansegg 1995: 112). Und mehr noch: »Demokratie ist nur Wirklichkeit, wo Politik als gewaltloser, geregelter Konflikt organisiert ist. Und umgekehrt: Die Institutionalisierung des gewaltlosen politischen Konfliktes ist gerade und nur in der Gestalt des demokratischen Verfassungsstaates gelungen.« (ebd.: 113)

\section{Tabelle 4: Grundwerte aus Sicht der Modalen Strukturierungstheorie}

\begin{tabular}{|c|c|c|}
\hline & $\begin{array}{l}\text { Sekundäre, instru- } \\
\text { mentelle Grund- } \\
\text { werte }\end{array}$ & $\begin{array}{l}\text { Primäre, terminale } \\
\text { Grundwerte }\end{array}$ \\
\hline $\begin{array}{l}\text { Strukturierung als } \\
\text { Ermöglichung } \\
\text { und Einschrän- } \\
\text { kung }\end{array}$ & Toleranz & $\begin{array}{l}\text { Freiheit } \\
\text { - negative } » \text { Freiheit von« Ein- } \\
\text { schränkungen } \\
\text { - positive »Freiheit zu« durch Er- } \\
\text { möglichungen }\end{array}$ \\
\hline $\begin{array}{l}\text { Strukturierung als } \\
\text { Differenzierung }\end{array}$ & Solidarität & $\begin{array}{l}\text { Gleichheit } \\
\text { - negative Gleichheit negativer } \\
\text { Freiheiten } \\
\text { - positive Gleichheit positiver Frei- } \\
\text { heiten } \\
\text { Gerechtigkeit } \\
\text { - gleich sichere gleiche Freiheiten }\end{array}$ \\
\hline Verzeitlichung & Frieden & $\begin{array}{l}\text { Sicherheit } \\
\text { - negative »Sicherheit vor« durch } \\
\text { erwartbare Abwesenheit von Ein- } \\
\text { schränkungen } \\
\text { - positive »Sicherheit durch« er- } \\
\text { wartbare Ermöglichungen } \\
\end{array}$ \\
\hline
\end{tabular}

Bobbio (1988: 33f.) nennt vier Ideale, nach denen zu leben ist, wenn Demokratie funktionieren soll: Toleranz, Gewaltfreiheit, freien Gedankenstreit 
und Lebensformenwandel sowie Brüderlichkeit. Er nennt also neben der freien Konkurrenz von Ideen und Veränderung von Lebensformen nichts anderes als: Toleranz, Frieden, Solidarität.

Diese Lebensideale finden sich denn auch in ethischen Betrachtungen. Im Anschluss an Arthur Schopenhauers Mitleidsethik z.B., lässt sich gut darstellen, wie Verletzen und Helfen mit sekundären Grundwerten zusammenhängen. In Schopenhauers Preisschrift über die Grundlage der Moral lassen sich vier Grundorientierungen ausmachen - er selber nennt ausdrücklich Egoismus, Mitleid, Bosheit (vgl. Fischer 2003: 111-116).

Tabelle 5: Vier Grundorientierungen in der Mitleidsethik von Schopenhauer

\begin{tabular}{|l|l|l|}
\hline $\begin{array}{l}\text { Positive und negative Selbst- } \\
\text { und Fremdorientierung }\end{array}$ & Eigenes & Fremdes \\
\hline Wohl & Egoismus & Mitleid \\
\hline Wehe & Masochismus & Bosheit \\
\hline
\end{tabular}

Die Orientierung des Handelns an Toleranz und Frieden, der Verzicht also auf negative Sanktion und Gewalt, verhindert Fremdschädigung, die Orientierung an Solidarität verhindert übertriebenen Egoismus (während ein >gesunder Egoismus`, d.h. wohlverstandene Selbstliebe, vermutlich Selbstschädigung verhindert). Ein respekt-, vertrauens- und liebevolles - echtes - Mitgefühl, das sich an Toleranz, Frieden und Solidarität ausrichtet, bildet ein Gegengift gegen Selbstsucht und Bosheit und wird dem Grundsatz der Schopenhauerschen Ethik »Verletze niemanden; vielmehr hilf allen, soweit du kannst« (Fischer 2003: 102) gerecht. Mit der Bosheit bringt Schopenhauer im Übrigen ins Spiel, dass die willentliche Negierung von Grundwerten bis zu lustvoller Schädigung, radikalem Exzess und kollektiver Auslöschung führen kann.

Alle Grundwerte müssen genauer interpretiert werden, insbesondere auf die jeweilige Qualität von Einschränkung und Ermöglichung hin. Bei genauerem Hinsehen nämlich zeigen sich auch sinnvolle Einschränkungen und unsinnige Ermöglichungen. Was hier aber Sinn oder keinen Sinn macht, kann nur entschieden werden, indem die Grundwerte inhaltlich genauer interpretiert werden. Dies hat Forst im Blick, wenn er fordert, Toleranz sei »mit Inhalt zu füllen« (2003: 49). Forst meint nun aber gar, erst dadurch werde sie zu »etwas Wertvollem«. Dessen Schluss daraus, Toleranz sei, da »normativ abhängig«, kein Wert, ist nicht zwingend. Es gibt nicht nur »falsche Toleranz«, d.h. allgemein intersubjektiv nicht zustimmungsfähig interpretierte und verwirklichte Toleranz, sondern ebenso falsche Solidarität, falschen Frieden, und dasselbe gilt für die primären Grundwerte. Ist Sterbehilfe ein solidarischer Akt? Ist der Verzicht auf Notwehr eine friedliche Handlung? Ist die Anerkennung menschenfeindlichen Handelns tolerant? Um welche Freiheit, Gleichheit, Sicherheit soll es gehen? Nur wohlverstandene Grundwerte sind ihrer Verwirklichung wert. Fraglich ist, ob falsch begriffe- 
ne Toleranz usw. überhaupt Toleranz usw. sei, aber auch, ob sich Grundwerte universell letztgültig interpretieren lassen.

Soviel zur Platzierung der demokratischen Grundwerte im ausgewählten gesellschaftstheoretischen Rahmen. Es fragt sich zum Schluss, ob der Begriff der Demokratie ausreicht, um den normativen Gehalt dieses Gesellschaftsbilds einzufangen. Welche konzeptuelle Alternative bietet sich an?

\section{Beteiligungsgesellschaft: RADIKALE PLURALE DEMOKRATISIERUNG AUS SICHT DER MODALEN STRUKTURIERUNGSTHEORIE}

Bruce Ackerman und Anne Alstott (2001) beschreiben in ihrem Buch zur Stakeholder-Gesellschaft ein, wie es im Untertitel heißt, »Modell für mehr Chancengleichheit«. Diese Idee greifen Gerd Grözinger, Michael Maschke und Claus Offe auf, um ihrerseits eine Teilhabegesellschaft zu entwerfen, in der ihr »Modell eines neuen Wohlfahrtsstaats« zur Entfaltung käme. Begründet wird dieses Modell deontologisch damit, dass in einer liberalen, dem »Grundsatz der gleichen realen Freiheit« (Grözinger/Maschke/Offe 2006: 17) folgenden Gesellschaft unter heutigen wirtschaftlichen Bedingungen die Einführung eines Grundeinkommens geboten scheint, sowie konsequentialistisch damit, dass die Einführung ökonomischer Bürgerrechte wünschenswert sei, da sie zur Lösung zentraler gesellschaftlicher Probleme (Bildungsferne, Erwerbslosigkeit, Kriminalität usw.) beitragen können. Die drei Autoren schlagen »den gesetzlichen Anspruch auf Auszahlung einer steuerfinanzierten >Sozialerbschaft«« (2006: 17) vor, da er dem Postulat gleicher Freiheit entspricht. Gleichheit wird hier zwar nicht nur minimalistisch als gleicher Schutz vor Diskriminierung bei der $\mathrm{Zu}$ - und Anerkennung von Rechten verstanden, aber auch nicht maximalistisch als Ergebnisgleichheit, sondern, so die mittlere Position, als Chancengleichheit. »Sie besagt etwa, dass alle Bürger durch nachweislich geeignete politisch-rechtliche Vorkehrungen in die Lage zu versetzen sind, von ihren Freiheitsrechten tatsächlich Gebrauch zu machen« (ebd.: 18). Dahinter steht die empirische These der Autoren, »dass die Entwicklung moderner Gesellschaften es mit sich bringt, dass der individuelle Freiheitsgebrauch immer voraussetzungsreicher wird und dass daher der Umfang der Vorkehrungen wächst, die im Interesse eines chancengleichen Zugangs zum Gebrauch von Freiheitsrechten erforderlich sind« (ebd.). Auch in Bezug auf die zweite Begriffshälfte "gleicher Freiheit« nehmen die drei Autoren eine mittlere Position ein: Freiheit wird nicht nur minimalistisch so aufgefasst, dass auch unter autoritärer Herrschaft noch zwischen Anpassung und Widerstand entschieden werden kann, aber auch nicht maximalistisch so, dass Bürger die Ordnung ihres Zusammenlebens gemeinsam und einvernehmlich, frei von Fremdherrschaft und Partikularinteresse bestimmen. Freiheit lässt sich vielmehr als »die 
Chance definieren, dass individuelle Bürger einen Lebensplan wählen und realisieren können, der einer von ihren Wünschen bestimmten Kombination von Lebensplänen und -tätigkeiten [...] entspricht« (ebd.: 20). Strukturierungstheoretisch gesagt: Es sollen erforderliche Ermöglichungen bestehen (und Einschränkungen ausbleiben), um Lebensziele und Lebensweise einander entsprechen zu lassen. Diese Vorstellung knüpft empirisch an den gängigen soziologischen Thesen der Differenzierung und Individualisierung an. Für eine der wichtigsten »Determinanten der Chancen des Freiheitsgebrauchs« halten die Autoren materielles Vermögen, dessen »soziale Funktion« sie im »Gewinn an Unabhängigkeit, Sicherheit und Gestaltungsfreiheit« (ebd.: 21) erkennen. Die individuelle Aussicht auf ein »Mehr an Freiheit« durch Vermögensbildung wirkt sehr leistungsmotivierend, darum scheint es den Autoren völlig verfehlt, die "privatautonome Verfügungsmacht über Kapital« abzuschaffen.

Die Teilhabegesellschaft rückt denn unter den Ermöglichungen die Lebenslage, genauer: die Mittel, und noch genauer: materielle Mittel, ins Zentrum der Aufmerksamkeit. Betrachtet man die Idee einer Teilhabegesellschaft indes aus der Perspektive der Demokratisierung der Demokratie und damit aus dem Blickwinkel förderlicher Voraussetzungen, erscheinen noch andere Mittel als bedeutsam. Mit Bourdieu (1983) gesprochen: Nicht nur ökonomisches, sondern ganz besonders kulturelles Kapital, aber auch soziales und schließlich symbolisches Kapital sind wichtig. Über Bourdieu hinaus können weitere Mittel in Betracht kommen, so ganz allgemein körperliche Gesundheit und verschiedene (motorische, perzeptive, kognitive, emotionale, volitive) Fähigkeiten (Husi 2010: 122f.). Eva Illouz (2009: 329ff.) fragt sich z.B., ob »emotionale Kompetenz« »eine neue Achse sozialer Schichtung« entstehen lässt. Teilhabe bedeutet, seinen Teil zu erhalten an den gesellschaftlich verfügbaren (materiellen, kulturellen, sozialen, personalen) Mitteln - und, wenn man sie auf die Lebenslage insgesamt bezieht, für seinen Teil von (sachlichen, sozialen, physischen, psychischen) Zwängen verschont zu bleiben. So weit reiche eigentlich die >Teilhabegesellschaft $<$. Wir leben indessen nicht nur in einer mehr oder weniger realisierten Teilhabegesellschaft, denn Zugehörigkeit realisiert sich nicht über Teilhabe, sondern über Teilnahme und, wie wir sagen könnten, Teilsein.

Im Teilsein begegnen sich Werte und Normen, fliessen Wünsche und Ziele sowie Rechte und Pflichten ineinander; kulturelle Verschiedenheit und institutionelle Normalität werden aufeinander abgestimmt, Authentizität und Richtigkeit austariert. Habermas‘ »Diskurstheorie der Moral« (1991: 7) und Forsts »kritische Theorie der Toleranz« (2003: 22) wollen mitunter das Verhältnis von ethischen Werten und moralischen Normen klären. Erstere alimentieren das Mögen (und indirekt das Wollen), letztere das Sollen und Dürfen. Wenn Theodor W. Adorno übrigens in 17 Vorlesungen Probleme der Moralphilosophie erörtert, verweist er gleich zu Beginn auf seine berühmte Sentenz in den Minima Moralia, wonach es kein richtiges Leben im falschen gebe, und findet, man könne »durchaus ein berechtigtes Interesse 
daran haben, etwas über das richtige Leben zu erfahren« (1996: 10). Er meidet jedoch »die verlogene Situation eines Gurus, eines Weisen« (ebd.: 11) ebenso wie einen »Kurzschluss zur Praxis« (ebd.). »Aber es ist so, dass, je ungewisser die Praxis geworden ist, je weniger wir tatsächlich wissen, was wir tun sollen, je weniger verbürgt uns ein richtiges Leben ist, wenn es denn je verbürgt gewesen sein sollte, dass dann um so hastiger danach gegriffen wird.« (ebd.: 12) Für Adorno liegt dem »Nichts-damit-anfangen-Können« die Abwertung des Denkens und der Intellektuellen sehr nahe. Zwar will Adorno nicht »Normen, Werte oder wie die grauslichen Wörter alle heissen mögen« (ebd.: 15) nennen, aber er sucht nach dem »Moment der Freiheit, ohne das so etwas wie richtiges Leben gar nicht gedacht werden kann« (ebd.: 16). Dieselbe Zweiheit wie Habermas und Forst nach ihm thematisiert Adorno, wenn er schließlich Moralphilosophie der Ethik vorzieht:

»Ethos $[\ldots]$ ist ein sehr schwer zu übersetzender Ausdruck, den man im allgemeinen [...] wiedergibt als Wesensart - also: wie einer ist, wie einer beschaffen ist. [...] Durch die Nivellierung der Problematik von Moral und Ethik wird von vornherein das entscheidende Problem der Moralphilosophie, nämlich das Verhältnis des einzelnen Individuums zu dem Allgemeinen, eskamotiert, es wird weggeschafft. Es steckt darin schon das: dass, wenn man nur seinem eigenen Ethos, seiner eigenen Beschaffenheit nach lebe - wenn man, wie man so schön sagt: sich selbst verwirkliche oder wie diese Phrasen alle lauten mögen -, dabei schon das richtige Leben herauskomme; was eine pure Illusion und eine pure Ideologie ist. Eine Ideologie im übrigen, die sich mit einer zweiten paart, nämlich mit der Ideologie, dass die Kultur und das Sichanpassen an die Kultur die Selbstveredelung, Selbstkultivierung des Individuums eigentlich dort leiste, wo die Kultur selbst gegenüber der Moralphilosophie zur Diskussion steht und eigentlich ein zu Kritisierendes wäre.« (ebd.: 23)

Adorno verwahrt sich, so können wir sagen, Normen, die zwischenmenschlich gelten, einfach auf Werte zu reduzieren, die persönlich gelten. Es würde nämlich in diesem Fall, so findet Adorno »die Vorstellung des richtigen Lebens, des richtigen Tuns darauf reduziert, dass man so handle, wie man ohnehin ist. Es wird also, indem man seinem Ethos, seiner Wesensbeschaffenheit nach handeln soll, das bloße So-Sein, dass man so und nicht anders >geartet` ist, zum Maßstab dessen gemacht, wie man sich verhalten soll« (ebd.: 26). Auf die Seite der Kultur schlagen mag sich Adorno jedoch auch nicht, denn er stellt »die Frage, ob die Kultur und das, wozu diese sogenannte Kultur geworden ist, überhaupt so etwas wie richtiges Leben zulässt oder ob sie ein Zusammenhang von Institutionen ist, der in zunehmendem Maß ein solches richtiges Leben geradezu verhindert« (ebd.: 28).

Wie das »Verhältnis zwischen dem Guten und dem Rechten«, das »Verhältnis zwischen dem attraktiven Charakter der Werte und dem obligatorischen Charakter der Normen« (Joas 1999: 258) genau zu konzipieren ist, wird kontrovers diskutiert (ebd.: 252-293). Hans Joas favorisiert im Anschluss an John Dewey und Charles Taylor den »Gedanken eines Reflexi- 
onsgleichgewichts zwischen dem Guten und dem Rechten« (ebd.: 264), der ihn schließlich Habermas ‘ Eintreten für einen »Primat des Rechten vor dem Guten« (ebd.: 274) kritisieren lässt:

»In der Situation des Handelns gibt es demnach keinen Primat des Guten oder des Rechten. Hier herrscht kein Über- oder Unterordnungsverhältnis, sondern eine Komplementarität. In der Situation des Handelns stoßen die irreduziblen Orientierungen in Richtung des Guten, die bereits in unseren Strebungen enthalten sind, auf die Prüfinstanz des Rechten. Was wir in diesen Situationen erreichen können, ist immer nur ein Reflexionsgleichgewicht zwischen unseren Orientierungen.« (ebd.: 270)

Es geht also um eine Balance zwischen mögen sowie sollen und dürfen. Für den >frühen und mittleren « Parsons, so dessen klassisch gewordene Ansicht, in der er Kants Idee der Freiheit als des Gehorsams gegenüber selbstgegebenen Gesetzen soziologisch wendet, »ist die symmetrische Beziehung zwischen der Autorität geltender Normen, denen der Aktor begegnet, und der in seiner Persönlichkeit verankerten Selbstkontrolle, ist die Entsprechung zwischen der Institutionalisierung und der Internalisierung von Werten wesentlich« (Habermas 1981: 310; vgl. auch Joas/Knöbl 2004: 96-106). Joas hält der bekannten theoretischen Position, dass Normen einfach Werte auf Situationen hin spezifizierten, »den idealistischen Fehlschluss einer Reduktion des Sozialen auf das Kulturelle« (1999: 273) vor. Stattdessen seien kulturelle Integration über Werte und soziale Integration über Normen klar zu unterscheiden - in der Begrifflichkeit der Modalen Strukturierungstheorie: kulturelle Differenzierung/Milieusstruktur und institutionelle Differenzierung/Lebensbereichestruktur.

»Die normative Regelung der sozialen Integration entstammt teilweise den kulturellen Werten; sie ist aber aus diesen nicht einfach abgeleitet, sondern resultiert aus einem Reflexionsgleichgewicht zwischen der Besinnung der kooperierenden Akteure auf ihr Zusammenwirken und den kulturellen Interpretationen. (Joas 1999: 273)

Joas argumentiert weiter, dass »der Gesichtspunkt des Rechten aber unvermeidlich in Konkurrenz tritt zu dem des Guten und seine Wirkung auf die Modifikation des Guten ausübt« (ebd.: 274). Auf diese Weise entfaltet sich also eine Dialektik von Werten und Normen.

Die zunächst subjektive Geltung von Werten bildet den Ausgangspunkt für die intersubjektive Geltung von Normen. Und in jeder Sozialisation prägen geltende Normen die Ausbildung eigener Werte mit. Demokratisch gefärbte regulative Sozialisationsbedingungen sind deshalb sehr bedeutsam. Sie helfen, den Geist des Demokratismus, die Orientierung an den Grundwerten zu verinnerlichen, und machen die Ausbildung von Autoritarismus (vgl. Vester 2003) unwahrscheinlich. Die Geltung demokratischer Mitbestimmungsregeln ist indes auf den kulturellen Nährboden förderlicher Grundwerte angewiesen. Teilsein bedeutet, dass sich persönliche Werte in 
legitimen Normen aufgehoben finden, ohne in ihnen restlos aufgehen zu müssen. Das Toleranzgebot schafft, in einem bestimmten Rahmen, Freiräume für abweichende Wertvorstellungen; liberales Zusammenleben pflegt möglichst weitgehend die paradoxe Norm der Nicht-Norm, d.h. des Normierungsverzichts oder zumindest schwacher Normierung - aber eben in einem bestimmten Rahmen bloß, denn (selbst- oder fremd-)schädliche oder egoistische Nonkonformität wird negativ sanktioniert. Eine besondere Herausforderung für jede liberale Gesellschaft ist die Tolerierung der Intoleranten, des Fundamentalismus unterschiedlicher Art, d.h. der performative Widerspruch im Freiheitsgebrauch der Menschen mit unfreiheitlicher Gesinnung, der für sich beansprucht, was diese ablehnt.

Nicht nur der - strukturbezogene - Aspekt des Teilseins erweitert die Perspektive einer Teilhabegesellschaft, sondern auch der - praxisbezogene Aspekt der Teilnahme. Teilnahme ist Einbezug in Handlungszusammenhänge, in gesellschaftliche Systeme, sei es in einer Leistungsrolle oder in einer Empfangsrolle. Der Blickwinkel der Demokratisierung lenkt den Blick besonders auf Möglichkeiten der Mitbestimmung, der Einwirkung auf Entscheidungen, deren Folgen einen selbst betreffen. Solche Partizipation weist verschiedene Stufen, Teilnahmequalitäten gleichsam, auf und reicht von Information über Mitwirkung (Mitsprache, Mitarbeit) und Mitentscheidung bis zu Selbstverantwortung (vgl. z.B. das Modell mitsamt Einflussgrößen von Lüttringhaus 2000: 72). Fühlt man sich schließlich als Teil zugehörig, wird wahrscheinlicher, dass man auch echt Anteil nimmt. Praxis bedeutet ja nicht nur zu handeln, sondern auch gleichzeitig zu erleben. Bezieht sich Teilnahme auf Handeln, so Anteilnahme auf Erleben. Anteilnahme ist von Mitgefühl getragen. Wer die Beteiligung anderer wahrnimmt, deren Perspektive einnimmt, sich in sie hineindenkt und vor allem einfühlt, nimmt Anteil. Die Erkenntnis der Wichtigkeit dieses Aspekts ist keineswegs neu: »Nach Hume bildet die Sympathie im Sinne der Fähigkeit, die Empfindungen anderer nachzuvollziehen, die Grundlage moralischen Urteilens« (Pauer-Studer 2003: 60). Neuere Diskussionen erörtern die »Rationalität des Gefühls«, so z.B. Ronald de Sousa in seinem so betitelten Buch. Wer partizipiert, entwickelt in der Regel ein Verantwortungsgefühl. Sodann finden sich zwischen Handeln und gefühlsmäßigem Erleben Entsprechungen:

- Sind soziale Beziehungen von tolerantem Handeln geprägt, stiftet dies im Erleben der Beteiligten ein Gefühl des Respekts. - Empfindet man Respekt, handelt man tolerant.

- Sind soziale Beziehungen von solidarischem Handeln geprägt, stiftet dies im Erleben der Beteiligten ein Gefühl der Liebe. - Empfindet man Liebe, handelt man solidarisch.

- Sind soziale Beziehungen von friedlichem Handeln geprägt, stiftet dies im Erleben der Beteiligten ein Gefühl des Vertrauens. - Empfindet man Vertrauen, handelt man friedlich. 
Anteilnahme nährt sich also vornehmlich von Respekt, Liebe und Vertrauen. Man empfindet mehr oder weniger Respekt, Liebe, Vertrauen und zeigt sie durch mehr oder weniger tolerantes, solidarisches, friedliches Tun und Lassen. Wenn Praxis Handeln und Erleben bedeutet, dann also mitunter Teilnahme und Anteilnahme. Wie der Gesichtspunkt des Teilseins und derjenige der Teilnahme weitet jener der Anteilnahme den Rahmen einer Teilhabegesellschaft aus.

Abbildung 3: Vier Aspekte von Beteiligung

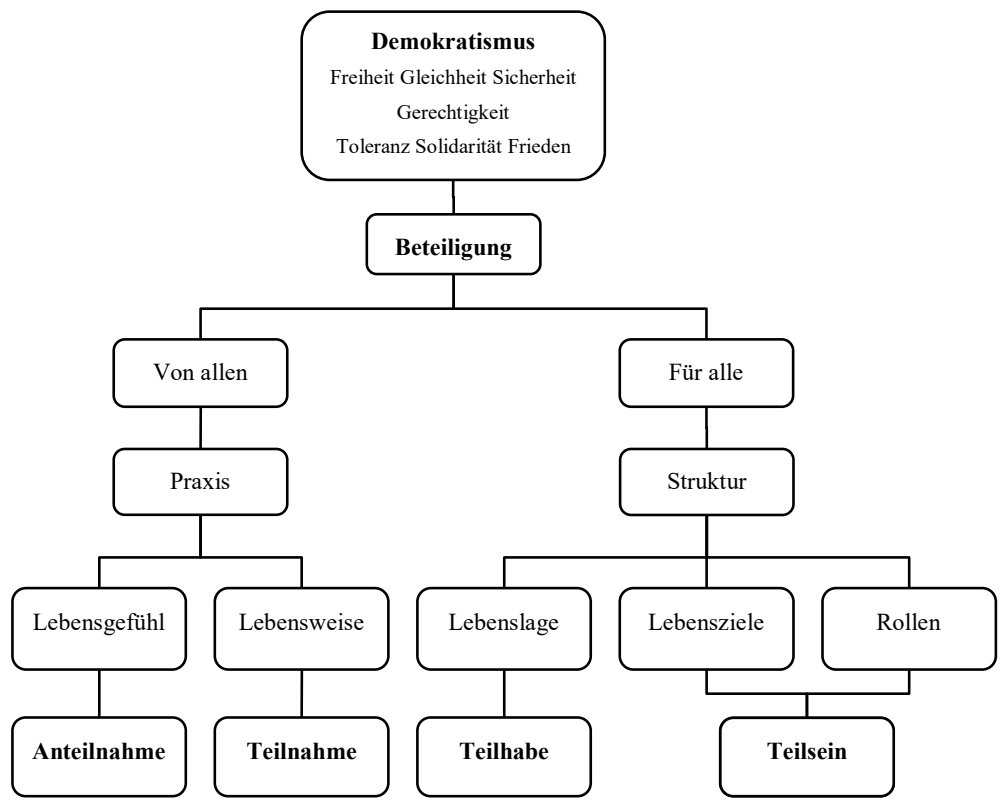

In diesen Formen: Teilhabe und Teilsein, Teilnahme und Anteilnahme, manifestiert sich Beteiligung. Menschen nehmen, haben, sind: Teil, und sie nehmen Anteil. Wer Teil hat, Teil ist, wer Teil und Anteil nimmt (bzw. erhält), ist beteiligt. Eine bekannte Redewendung gilt hier ganz besonders: Demokratie (und ihre Demokratisierung) gelingt in dem Masse, wie Betroffene zu Beteiligten gemacht werden. An dieser Stelle soll nun das gesellschaftstheoretisch fundierte und normativ gehaltvolle Konzept der Beteiligungsgesellschaft eingeführt werden. Eine Beteiligungsgesellschaft - jenseits der überlieferten Wortbedeutung einer rechtlich geregelten Organisationsform natürlich - realisiert sich, insoweit sie auf gerechte Weise für alle Menschen, gegenwärtig und auch künftig, erwartbar Teilhabe und Teilsein, Teilnahme und Anteilnahme gewährleistet. Der Geist des Demokratismus verwirklicht sich mehr oder minder in einer Beteiligungsgesellschaft. Sie steht nicht im Spannungsfeld von >nicht mehr und >noch nicht $<$ - sie ist schon und wird noch, sie besteht bereits und ist, als offene Gesellschaft, be- 
reit für eine vielgestaltige weitergehende Demokratisierung. Sie stellt ein unvollendetes Projekt dar, das auch nicht vollendbar scheint. Insofern sich eine Gesellschaft radikal und plural im oben geschilderten Sinne demokratisiert, entwickelt sie sich zu einer Beteiligungsgesellschaft. Wenn die Moderne ein immer noch »unvollendetes Projekt» darstellt, wie Habermas (1981a) einst formulierte, dann hat dies wesentlich damit zu tun, dass weiterhin oder neuerdings wichtige Demokratisierungsdefizite bestehen und Demokratisierungspotenziale nicht ausgeschöpft werden. Dem Gedanken an Grade erreichter Demokratisierung entspricht, dass sich eine Beteiligungsgesellschaft in unterschiedlichem Ausmaß herausbilden kann. Die Begriffe der Demokratie und der Beteiligungsgesellschaft bilden keine Synonyme, da sich der Begriff der Demokratie nur auf die von demokratischen Regeln geprägten Institutionen (verschiedener Lebensbereiche) bezieht, der Begriff der Beteiligungsgesellschaft aber auf die Gesellschaft insgesamt, d.h. auf die gesellschaftliche Praxis und Struktur. In einer Beteiligungsgesellschaft sind allen Gesellschaftsmitgliedern Teilhabe und Teilsein, Teilnahme und Anteilnahme gegeben. Den Überflüssigen (Bude/Willisch 2008) mangelt es dagegen weitgehend an Teilnahme und Anteilnahme und infolgedessen an Teilhabe und Teilsein, während andere davon im Überfluss genießen.

Demokratisierung in der doppelten Bedeutung der Institutionalisierung demokratischer Regeln (inner- und außerhalb der Politik) sowie der Schaffung gerechter günstiger Voraussetzungen für deren Entwicklung und praktische Geltung verweist also auf Beteiligung im umfassenden Sinne. Praxis im Geiste des Demokratismus bedeutet nicht nur einfach mitzubestimmen, sondern nach bestem Wissen und Gewissen sich wie andere zu beteiligen, d.h. durch sie, die Praxis, zugleich Teilhabe und Teilsein, Teilnahme und Anteilnahme zu realisieren. Demokratische Mitbestimmungsrechte bedürfen der Unterstützung durch Mitbestimmungsmittel und Mitbestimmungswünsche, damit Mitbestimmung faktisch realisiert wird: Die Gesellschaftsstruktur, in der individuelle Lebenslagen, Lebensziele und Rollen ihren Platz haben, enthält wichtige Voraussetzungen für eine lebendige Demokratie, insbesondere die verbreitete Verinnerlichung demokratischer Grundwerte jenseits aller kulturellen Differenzierung. Infolgedessen finden sich demokratische Gesinnungen in allen Milieus, und, in Bezug auf die hierarchische Differenzierung, die hinreichende (Gleich-)Verteilung von Machtmitteln. Gewiss kommt Bildung unter diesen Mitteln eine besondere Bedeutung zu: Nicht allein Wissen und kognitive Fähigkeiten sind hierbei wichtig, sondern ebenso, denkt man an die Anteilnahme, die sogenannte >Herzensbildung〈, emotionale Fähigkeiten also. Eine Reflexion der Herzensbildung kann, nebenbei gesagt, auf die gefühls- und mitleidsethische Tradition zurückgreifen, die besonders von David Hume und Adam Smith, Jean-Jacques Rousseau und Arthur Schopenhauer geprägt worden ist; die neuere Debatte entfaltet sich um den Begriff der »emotionalen Intelligenz« von Daniel Goleman. Was die institutionelle Differenzierung betrifft, so sollten andere Regeln nicht der Geltung demokratischer Regeln zuwiderlaufen. Wenn man 
das, was Max Weber, wie am Anfang erwähnt, mit seiner wertetheoretischen Grundlegung im Sinn hatte, durch die Brille der Beteiligung im dargestellten Sinne betrachtet, gelangt also Folgendes in den Blick:

- Ihre Wertewirklichkeit haben demokratische Grundwerte (Geist des Demokratismus) in den Lebenszielen der Individuen, das demokratische Imaginäre konkretisiert sich in Wertinterpretationen.

- Werteverwirklichung (Demokratisierung) vollzieht sich in der und durch die Praxis: Institutionalisieren demokratischer Mitbestimmungsregeln sowie Schaffen günstiger Demokratiebedingungen.

- Die Werteverwirklichtheit (Beteiligung) manifestiert sich in Praxis und Struktur.

- Praxis umfasst Teilnahme und Anteilnahme und bedeutet mitunter, freiheits-, gleichheits-, sicherheitsförderlich zu handeln und daher andere tolerant, solidarisch, friedlich zu behandeln; diesem Tun und Lassen entspricht respekt-, liebe- und vertrauensvolles Erleben.

- Struktur umfasst Teilhabe und Teilsein und enthält Freiheiten, Gleichheiten, Sicherheiten, und dies bedeutet, als gerechte: gleich sichere gleiche negative Freiheiten von Einschränkung und positive Freiheiten der Ermöglichung.

Dies alles umfasst Zusammenleben im Geiste des Demokratismus, die Verwirklichung demokratischer Werte in der Beteiligungsgesellschaft.

\section{Tabelle 6: Grundwerte in der Beteiligungsgesellschaft}

\begin{tabular}{|c|c|c|}
\hline \multicolumn{3}{|c|}{$\begin{array}{c}\text { STRUKTUR } \\
\text { gleich sichere gleiche negative Freiheit von Einschränkung und positive Freiheit der } \\
\text { Ermöglichung }\end{array}$} \\
\hline TEILHABE & \multicolumn{2}{|c|}{ TEILSEIN } \\
\hline $\begin{array}{c}\text { hierarchische } \\
\text { Differenzierung }\end{array}$ & $\begin{array}{c}\text { institutionelle } \\
\text { Differenzierung }\end{array}$ & $\begin{array}{c}\text { kulturelle } \\
\text { Differenzierung }\end{array}$ \\
\hline $\begin{array}{l}\text { distributive Gerechtigkeit: } \\
\text { - gerechte Verteilung von } \\
\text { Mitteln \& Zwängen }\end{array}$ & $\begin{array}{l}\text { regulative Gerechtigkeit: } \\
\text { - bürgerliche, politische, } \\
\text { soziale Rechte } \\
\text { - gerechte außerpolitische } \\
\text { Mitbestimmungsregeln/ } \\
\text { Rollenverteilung }\end{array}$ & $\begin{array}{l}\text { verinnerlichte Grund- } \\
\text { werte }\end{array}$ \\
\hline \multicolumn{3}{|c|}{$\begin{array}{c}\text { PRAXIS } \\
\text { beteiligtes und beteiligendes Handeln und Erleben }\end{array}$} \\
\hline TEILNAHME & & ANTEILNAHME \\
\hline $\begin{array}{l}\text { demokratische politi- } \\
\text { sche und außerpoliti- } \\
\text { sche Mitbestimmung } \\
\text { - tolerantes, solidari- } \\
\text { sches, friedliches Han- } \\
\text { deln }\end{array}$ & $\begin{array}{l}\text { gesellschaftlicher } \\
\text { Zusammenhalt }\end{array}$ & $\begin{array}{l}\text { - verantwortungsvolles } \\
\text { Erleben } \\
\text { - respekt-, liebe-, und } \\
\text { vertrauensvolles Erle- } \\
\text { ben }\end{array}$ \\
\hline
\end{tabular}


Das Konzept der Beteiligungsgesellschaft gibt im Übrigen auch Antworten auf die Fragen, was die Gesellschaft zusammenhält und was sie auseinander treibt (Heitmeyer 1997a, 1997b). Soziale Kohäsion bzw. gesellschaftliche Integration (vgl. auch Husi 2010; Chiesi 2005) ergibt sich unmittelbar aus tolerantem, solidarischem, friedlichem Zusammenleben. Im Sinne von Giddens' Theorem der Dualität von Struktur dienen gesellschaftsstrukturell verwirklichte Freiheiten, Gleichheiten und Sicherheiten medial an jenen Werten orientiertem Handeln. Und umgekehrt wird eine solche Gesellschaftsstruktur ihrerseits durch eine Praxis hervorgebracht und reproduziert, die Toleranz, Solidarität und Frieden kennzeichnen. Gesellschaftlicher $\mathrm{Zu}$ sammenhalt gründet unmittelbar auf Teilnahme und Anteilnahme und erweist sich in einer Praxis, die geprägt ist von tolerantem, solidarischem, friedlichem Handeln sowie respektvollem, liebevollem, vertrauensvollem Erleben.

Gelingendes Leben in einer Beteiligungsgesellschaft will gelernt sein. Ein erstes wichtiges Lernfeld ist bereits die Herkunftsfamilie. »Die Frage nach dem Geist der Demokratie verschiebt sich damit zu der Frage, wo und wie in einer Gesellschaft Freiheiten aus- und eingeübt werden können«, schreibt z.B. Beck (1997: 204) zur Demokratisierung der Familie. »Demokratie ist die einzige politisch verfasste Gesellschaftsordnung, die gelernt werden muss«. Was Negt (2008: 41) hier formuliert, gilt für die Beteiligungsgesellschaft insgesamt. »Demokratie macht Lernen notwendig; ohne Mitbestimmung in allen Lebensbereichen, die wichtige Angelegenheiten der Menschen regulieren, ist demokratisches Lernen nicht möglich« (Negt 2008: 41). D.h. also nicht nur: Demokratie macht Lernen notwendig; sondern auch: Lernen macht Demokratie notwendig. Zu erlernen ist die Kunst des Zusammenlebens. Das zur Beteiligungsgesellschaft gehörige Paradigma individuell-sozialen Lebens ist weniger dasjenige der Lebenskunst (Schmid 1998) als jenes der Zusammenlebenskunst. Die radikale und plurale Demokratie (mitsamt ihren Voraussetzungen) wäre nichts anderes als eine weitreichend verwirklichte Beteiligungsgesellschaft in der Zusammenlebenskünstler und -künstlerinnen lebten. Zusammenlebenskunst erweist sich darin, dass Menschen sich und andere am Zusammenleben beteiligen und dafür günstige Lebensbedingungen schaffen - frei nach Joseph Beuys: jeder Mensch eine Zusammenlebenskünstlerin, ein Zusammenlebenskünstler! Der Mensch, der die radikale und plurale Demokratie belebt, ist ein vielseitiger, achtsamer, kreativer Zusammenlebenskünstler. Es handelt sich letztlich um ein gemeinsames Erlernen der Zusammenlebenskunst - wo Einsamkeit war, soll Gemeinsamkeit werden. Demokratisierung, die Entwicklung zur Beteiligungsgesellschaft ist »auf längere Sicht als ein sich selbst korrigierender Lernprozess« (Habermas 2001: 144) zu verstehen. Sie zehrt von Großprojekten sozialer Bewegungen und in Organisationen ebenso wie von den unzähligen Kleinprojekten in Familien und anderen Gruppen, Freundschaften usw. Die Entwicklung zur Beteiligungsgesellschaft ist Alltagsdemokratisierung. 
Fassen wir zusammen: Als Grundwerte der Moderne kristallisieren sich während der demokratischen Revolution primär Freiheit, Gleichheit und Sicherheit heraus, die sich in einem anspruchsvollen Konzept der Gerechtigkeit vereinen lassen, und sekundär Toleranz, Solidarität und Frieden. All diese Werte bilden den Geist des Demokratismus und werden in Prozessen der Demokratisierung verwirklicht. Während Demokratisierung im engen Sinne zunächst in der Politik und dann in vielen anderen Lebensbereichen demokratische Regeln institutionalisiert, schafft Demokratisierung im weiten Sinne günstige strukturelle Bedingungen dafür. In der Orientierung an Gleichheit demokratisiert sich eine Gesellschaft radikal, in der Orientierung an Freiheit plural. Fokussieren Diagnoseinstrumente wie das Demokratiebarometer angesichts skeptischer empirischer Einschätzungen (Ende der Demokratie, Postdemokratie) auf die politischen Institutionen, so erfordert ein umfassendes Demokratisierungsverständnis, das auf Alltagsdemokratie abzielt, ein entsprechendes Gesellschaftsbild. Vor allem von Giddens (und Bourdieu) ausgehend lässt sich diesem Erfordernis mit einer Modalen Strukturierungstheorie begegnen, mit der die Weltbezüge der Grundwerte sichtbar gemacht werden können. Strukturierung (Ermöglichung und Einschränkung), Differenzierung und Verzeitlichung sind dabei wichtigste Aspekte. Diese theoretische Konzeption ist normativ gehaltvoll, indem sie eine Beteiligungsgesellschaft entwerfen und anstreben lässt. Beteiligung meint darin Teilhabe plus Teilsein plus Teilnahme plus Anteilnahme. Moderne Gesellschaften haben sich seit längerem auf den Weg zur Beteiligungsgesellschaft gemacht. An ein Ende des Wegs können sie nicht gelangen, da es sich um ein offenes Konzept handelt, das sich stetig selber vielfältig modernisiert. Die Beteiligungsgesellschaft bleibt ein unvollendetes Projekt wie ein unvollendbares Projekt. Zusammenlebenskünstlerinnen und Zusammenlebenskünstler, die fähig und willens sind, Beteiligung in mannigfaltiger Hinsicht $\mathrm{zu}$ realisieren, bringen eine Beteiligungsgesellschaft voran. Beteiligungsgesellschaft und Zusammenlebenskunst sind gesellschafts- und individuumsbezogene Utopien, die ihren Ort zur Entfaltung nicht im Nirgendwo, sondern in den real existierenden demokratischen Gesellschaften und individuellen Lebensentwürfen haben. Ihnen gehört nach dem Ende der Demokratie - die Zukunft der Demokratie.

\section{LITERATUR}

Ackerman, Bruce/Alstott, Anne (2001): Die Stakeholder-Gesellschaft. Ein Modell für mehr Chancengleichheit, Frankfurt a.M./New York: Campus.

Adorno, Theodor W. (1996): Probleme der Moralphilosophie (1963) (Nachgelassene Schriften Abteilung IV: Vorlesungen, Bd. 10), Frankfurt a.M.: Suhrkamp.

Beck, Ulrich (1997): »Demokratisierung der Familie«, in: Ders. (Hg.): Kinder der Freiheit, Frankfurt a.M.: Suhrkamp, S. 195-216. 
Beyme, Klaus von (1994): Systemwechsel in Osteuropa, Frankfurt a.M.: Suhrkamp.

Bobbio, Norberto (1988): »Die Zukunft der Demokratie«, in: Ders., Die Zukunft der Demokratie, Berlin: Rotbuch, S. 7-34.

Bourdieu, Pierre (1979): Entwurf einer Theorie der Praxis. Auf der ethnologischen Grundlage der kabylischen Gesellschaft, Frankfurt a.M.: Suhrkamp.

Bourdieu, Pierre (1983): »Ökonomisches Kapital, kulturelles Kapital, soziales Kapital«, in: Reinhard Kreckel (Hg.): Soziale Ungleichheiten ( = Soziale Welt Sonderband 2), Göttingen: Schwartz, S. 183-198.

Bourdieu, Pierre (1987): Sozialer Sinn. Kritik der theoretischen Vernunft, Frankfurt a.M.: Suhrkamp.

Brandt, Willy (1973): »Die Alternative«, in: Martin Greiffenhagen (Hg.): Demokratisierung in Staat und Gesellschaft, München: Piper, S. 45-46.

Brandt, Willy (1979): »28. Oktober 1969 Bundeskanzler Willy Brandt«, in: Klaus von Beyme (Hg.): Die großen Regierungserklärungen der deutschen Bundeskanzler von Adenauer bis Schmidt, München/Wien: Hanser, S. 251-281.

Bude, Heinz/Willisch, Andreas (Hg.) (2008): Exklusion. Die Debatte über die >Überflüssigen`, Frankfurt a.M.: Suhrkamp.

Bühlmann, Marc/Merkel, Wolfgang/Müller, Lisa/Giebler, Heiko/Weßels, Bernhard (2011): Demokratiebarometer - ein neues Instrument zur Messung von Demokratiequalität. Siehe http://www.democracybarometer.org/baroapp/files/flh/gij/yrl/Demokratiebarometer_Konzept.pdf (erscheint in: Zeitschrift für vergleichende Politikwissenschaft).

Chiesi, Antonio M. (2005): »Soziale Kohäsion und verwandte Konzepte«, in: Nikolai Genov (Hg.): Die Entwicklung des soziologischen Wissens. Ergebnisse eines halben Jahrhunderts, Wiesbaden: VS Verlag, S. 239256.

Crouch, Colin (2008): Postdemokratie, Frankfurt a.M.: Suhrkamp.

Fischer, Peter (2003): Einführung in die Ethik, München: Fink.

Forst, Rainer (2003): Toleranz im Konflikt. Geschichte, Gehalt und Gegenwart eines umstrittenen Begriffs, Frankfurt a.M.: Suhrkamp.

Fraser, Nancy (2001): Die halbierte Gerechtigkeit. Schlüsselbegriffe des postindustriellen Sozialstaats, Frankfurt a.M.: Suhrkamp.

Fraser, Nancy (2003): »Soziale Gerechtigkeit im Zeitalter der Identitätspolitik«, in: Nancy Fraser/Axel Honneth: Umverteilung oder Anerkennung? Eine politisch-philosophische Kontroverse, Frankfurt a.M.: Suhrkamp, S. 13-128.

Giddens, Anthony (1984): Interpretative Soziologie. Eine kritische Einführung, Frankfurt a.M.New York: Campus.

Giddens, Anthony (1988): Die Konstitution der Gesellschaft, Frankfurt a.M./New York: Campus. 
Giddens, Anthony (2001): »Demokratie«, in: Ders.: Entfesselte Welt. Wie die Globalisierung unser Leben verändert, Frankfurt a.M.: Suhrkamp, S. 87-103.

Greiffenhagen, Martin (1973): »Einleitung«, in: Ders. (Hg.): Demokratisierung in Staat und Gesellschaft, München: Piper, S. 11-41.

Grözinger, Gerd/Maschke, Michael/Offe, Claus (2006): Die Teilhabegesellschaft. Modell eines neuen Wohlfahrtsstaats, Frankfurt a.M./New York: Campus.

Guéhenno, Jean-Marie (1994): Das Ende der Demokratie, München/Zürich: Artemis \& Winkler.

Habermas, Jürgen (1981): Theorie des kommunikativen Handelns. Bd. 2: Zur Kritik der funktionalistischen Vernunft, Frankfurt a.M.: Suhrkamp.

Habermas, Jürgen (1981a): »Die Moderne - ein unvollendetes Projekt« (1980), in: Ders., Kleine Politische Schriften I-IV, Frankfurt a.M.: Suhrkamp, S. 444-464.

Habermas, Jürgen (1991): Erläuterungen zur Diskursethik, Frankfurt a.M.: Suhrkamp.

Habermas, Jürgen (2001): »Der demokratische Rechtsstaat - eine paradoxe Verbindung widersprüchlicher Prinzipien?«, in: Ders.: Zeit der Übergänge, Frankfurt a.M.: Suhrkamp, S. 133-151.

Habermas, Jürgen (2004): »Ist die Herausbildung einer europäischen Identität nötig, und ist sie möglich?«, in: Ders.: Der gespaltene Westen. Kleine Politische Schriften X, Frankfurt a.M.: Suhrkamp, S. 68-82.

Heitmeyer, Wilhelm (Hg.) (1997a): Was hält die Gesellschaft zusammen?, Frankfurt a.M.: Suhrkamp.

Heitmeyer, Wilhelm (Hg.) (1997b): Was treibt die Gesellschaft auseinander?, Frankfurt a.M.: Suhrkamp.

Huntington, Samuel P. (1991): The Third Wave. Democratization in the Late Twentieth Century, Norman/London: University of Oklahoma.

Husi, Gregor (2010): »Die Soziokulturelle Animation aus strukturierungstheoretischer Sicht«, in: Bernard Wandeler (Hg.): Soziokulturelle Animation. Professionelles Handeln zur Förderung von Zivilgesellschaft, Partizipation und Kohäsion, Luzern: Interact, S. 97-155.

Husi, Gregor/Meier Kressig, Marcel (1998): Der Geist des Demokratismus. Modernisierung als Verwirklichung von Freiheit, Gleichheit und Sicherheit, Münster: Westfälisches Dampfboot.

Illouz, Eva (2009): Die Errettung der modernen Seele. Therapien, Gefühle und die Kultur der Selbsthilfe, Frankfurt a.M.: Suhrkamp.

Joas, Hans (1999): Die Entstehung der Werte, Frankfurt a.M.: Suhrkamp.

Joas, Hans/Knöbl, Wolfgang (2004): Sozialtheorie. Zwanzig einführende Vorlesungen, Frankfurt a.M.: Suhrkamp.

Jörke, Dirk (2005): »Auf dem Weg zur Postdemokratie«, in: Leviathan 33, S. 482-491. 
Kielmansegg, Peter Graf (1995): »Frieden durch Demokratie«, in: Dieter Senghaas (Hg.): Den Frieden denken. Si vis pacem, para pacem, Frankfurt a.M.: Suhrkamp, S. 106-123.

Laclau, Ernesto/Mouffe, Chantal (1991): Hegemonie und radikale Demokratie. Zur Dekonstruktion des Marxismus, Wien: Passagen.

Lessenich, Stephan (2010): »Soziologie der Sozialpolitik«, in: Georg Kneer/Markus Schroer (Hg.): Handbuch Spezielle Soziologien, Wiesbaden: VS Verlag für Sozialwissenschaften, S. 555-568.

Luhmann, Niklas (1993): Gibt es in unserer Gesellschaft noch unverzichtbare Normen?, Heidelberg: Müller.

Lüttringhaus, Maria (2000): Stadtentwicklung und Partizipation. Fallstudien aus Essen Katernberg und der Dresdner Äußeren Neustadt, Bonn: Stiftung Mitarbeit.

Mannheim, Karl (1958): Mensch und Gesellschaft im Zeitalter des Umbaus, Darmstadt: Gentner.

Marshall, Thomas H. (1992): »Staatsbürgerrechte und soziale Klassen«, in: Ders.: Bürgerrechte und soziale Klassen. Zur Soziologie des Wohlfahrtsstaates, Frankfurt a.M./New York: Campus, S. 33-94.

Martens, Helmut (2010): Neue Wirtschaftsdemokratie. Anknüpfungspunkte im Zeichen der Krise von Ökonomie, Ökologie und Politik, Hamburg: VSA.

Marti, Urs (2006): Demokratie. Das uneingelöste Versprechen, Zürich: Rotpunktverlag.

Marx, Karl/Engels, Friedrich (1959): »Manifest der Kommunistischen Partei«, in: MEW Bd. 4, Berlin: Dietz, S. 459-493.

Merkel, Wolfgang (2003): »)Eingebettete< und defekte Demokratien: Theorie und Empirie«, in: Claus Offe (Hg.): Demokratisierung der Demokratie. Diagnosen und Reformvorschläge, Frankfurt a.M./New York: Campus, S. 43-71.

Meyer, Thomas (2005): Theorie der Sozialen Demokratie, Wiesbaden: VS Verlag.

Müller, Hans-Peter (2005): »Handeln und Struktur. Pierre Bourdieus Praxeologie«, in: Catherine Colliot-Thélène/Etienne François/Gunter Gebauer (Hg.): Pierre Bourdieu: Deutsch-französische Perspektiven, Frankfurt a.M.: Suhrkamp, S. 21-42.

Negt, Oskar (2008): »Demokratie als Lebensform«, in: Neue Gesellschaft Frankfurter Hefte 55, S. 37-41.

Negt, Oskar (2010): Der politische Mensch. Demokratie als Lebensform, Göttingen: Steidl.

Offe, Claus (Hg.) (2003): Demokratisierung der Demokratie. Diagnosen und Reformvorschläge, Frankfurt a.M./New York: Campus.

Parsons, Talcott (1970): Das System moderner Gesellschaften, Weinheim/München: Juventa.

Pauer-Studer, Herlinde (2003): Einführung in die Ethik, Wien: WUV. 
Perthes, Volker (2011): Der Aufstand. Die arabische Revolution und ihre Folgen, München: Pantheon.

Reckwitz, Andreas (2003): »Grundelemente einer Theorie sozialer Praktiken. Eine sozialtheoretische Perspektive«, in: Zeitschrift für Soziologie 32, S. 282-301.

Rokeach, Milton (1973): The Nature of Human Values, New York: The Free Press.

Schäfer, Reinhild (2001): Demokratisierung der Geschlechterverhältnisse. Die politischen Strategien der neuen Frauenbewegung gegen Gewalt, Bielefeld: Kleine.

Schimank, Uwe (2000): Handeln und Strukturen. Einführung in die akteurtheoretische Soziologie, Weinheim/München: Juventa.

Schmid, Wilhelm (1998): Philosophie der Lebenskunst. Eine Grundlegung, Frankfurt a.M.: Suhrkamp.

Schmidt, Manfred G. (Hg.) (2010): Wörterbuch zur Politik, (3., überarb. u. aktual. Aufl.), Stuttgart: Kröner.

Sedmak, Clemens (2010): »Europäische Grundwerte, Werte in Europa: Einleitung zum Gesamtprojekt«, in: Ders. (Hg.): Solidarität. Vom Wert der Gemeinschaft (Grundwerte Europas, Bd. 1), Darmstadt: Wissenschaftliche Buchgesellschaft, S. 9-42.

Singelnstein, Tobias/Stolle, Peer (2006): Die Sicherheitsgesellschaft. Soziale Kontrolle im 21. Jahrhundert, Wiesbaden: VS Verlag.

Stein, Ekkehart (1995): Demokratisierung der Marktwirtschaft, BadenBaden: Nomos.

Truhlar, Dalibor (2006): Demokratismus. Philosophie der demokratischen Weltanschauung, Frankfurt a.M.: Lang.

Vereinte Nationen (2000): Millenniums-Erklärung der Vereinten Nationen. Generalversammlungsresolution 55/2 vom 8. September 2000. Siehe http://www.unric.org/html/german/mdg/millenniumerklaerung.pdf

Vester, Michael (2003): »Autoritarismus und Klassenzugehörigkeit«, in: Alex Demirovic (Hg.): Modelle kritischer Gesellschaftstheorie. Traditionen und Perspektiven der Kritischen Theorie, Stuttgart/Weimar: Metzler, S. 195-224.

Vilmar, Fritz (1973): Strategien der Demokratisierung. Bd. 1: Theorie der Praxis, Darmstadt/Neuwied: Luchterhand.

Vilmar, Fritz (1973a): Strategien der Demokratisierung. Bd. 2: Modelle und Kämpfe der Praxis, Darmstadt/Neuwied: Luchterhand.

Vorländer, Hans (2003): Demokratie. Geschichte, Formen, Theorien, München: Beck.

Wildt, Andreas (1998): »Solidarität - Begriffsgeschichte und Definition heute«, in: Kurt Bayertz (Hg.): Solidarität. Begriff und Problem, Frankfurt a.M.: Suhrkamp, S. 202-216.

Zoll, Rainer (2000): Was ist Solidarität heute?, Frankfurt a.M.: Suhrkamp. 